\title{
Cancers du pancréas exocrine
}

\author{
U. Duran, D. Brisbois, R. Materne, C. Tchuisse Noukoua, N. Blétard, E. Mutijima,
} A. Nchimi

Les tumeurs du pancréas exocrine sont fréquentes et souvent létales. Sur le plan histologique, I'adénocarcinome canalaire en est le type le plus fréquent. Cet article décrit les signes cliniques, biologiques et radiologiques, ainsi que la contribution respective de ceux-ci au diagnostic et à la prise en charge des tumeurs du pancréas exocrine. Un algorithme décisionnel prenant en compte la valeur diagnostique et le coût de chaque technique dans l'évaluation des cancers pancréatiques est discuté. Une mention spéciale est accordée à l'utilisation optimale des progrès en imagerie médicale, ainsi que leurs bénéfices escomptés. Une bonne connaissance de la sémiologie radiologique permet actuellement un pronostic de résécabilité précis des tumeurs pancréatiques. L'impact des progrès de l'imagerie sur la courbe de mortalité des cancers du pancréas exocrine demeure cependant modeste. En effet, la mortalité des cancers du pancréas exocrine dépend essentiellement de la résécabilité des tumeurs. Cette dernière est souvent proportionnelle à la taille de la tumeur à sa découverte. Pour diverses raisons incluant la situation anatomique du pancréas, les signes cliniques susceptibles de justifier le recours à l'imagerie à la recherche d'un cancer du pancréas manquent de précision diagnostique, tandis que les seules indications d'imagerie chez les sujets asymptomatiques sont les risques génétiques élevés. L'amélioration de l'estimation du risque de cancer du pancréas sur la base d'analyses biologiques, combinée aux bonnes valeurs diagnostiques des techniques d'imagerie, pourraient donc in fine faire reculer la mortalité des cancers du pancréas.

(c) 2015 Elsevier Masson SAS. Tous droits réservés.

Mots-clés : Cancers; Résécabilité; Cancers exocrines; Adénocarcinome; Pancréas; Diagnostic

\section{Plan}

\section{- Introduction}

1

- Considérations techniques en imagerie du pancréas Échographie

Tomodensitométrie

Imagerie par résonance magnétique

Tomographie par émission de positrons

- Éléments sémiologiques en imagerie pancréatique Anomalies de la trophicité

Voies biliaires et canaux pancréatiques

Changement des propriétés tissulaires

Envahissement des structures locorégionales

- Types histologiques

Adénocarcinome canalaire et variants $\quad 11$

Autres tumeurs du pancréas exocrine $\quad 13$

- Diagnostic différentiel $\quad 14$

Affections tumorales $\quad 15$

Affections non tumorales $\quad 16$

- Conclusion

\section{Introduction}

L'incidence du cancer du pancréas se classe à la treizième place chez l'homme. Sa mortalité en est la huitième cause par cancer sur l'ensemble du globe, et la quatrième dans les pays développés ${ }^{[1]}$. En Europe, le nombre annuel moyen de décès varie actuellement entre cinq et dix par 100000 habitants. Il semble avoir augmenté dans tous les pays depuis les années 1950 jusqu'à la fin des années 1980 pour les hommes, et des années 1990 pour les femmes ${ }^{[2]}$. Cette stabilisation est mise en rapport avec la maîtrise des facteurs carcinogènes connus, au premier rang desquels le tabagisme ${ }^{[2]}$. Malgré une avancée technologique sans cesse croissante de l'imagerie, aucun effet bénéfique direct n'a été démontré jusqu'alors sur la survie des patients. Ce bénéfice potentiel peut cependant être anticipé, dans la mesure où les méthodes actuelles d'imagerie permettent de détecter des tumeurs de petite taille (moins de $2 \mathrm{~cm}$ ) dont le pronostic est meilleur que celui des tumeurs classiquement découvertes à un stade avancé ${ }^{[3]}$. Quelques difficultés stratégiques et diagnostiques restent néanmoins bien présentes sur la voie d'un infléchissement de la courbe de mortalité du cancer du pancréas. Elles incluent d'une part la problématique que représente la sélection 
de groupes de personnes à dépister, au sein de laquelle le gain en termes de survie serait réel, et d'autre part la difficulté iconographique à différencier les tumeurs du pancréas de certaines anomalies non tumorales. Il est ainsi impératif que les patients avec une prédisposition génétique, sous la forme d'un syndrome de cancers héréditaires ou d'une mutation génétique déterminée, qui représentent environ $10 \%$ des patients avec un cancer du pancréas ${ }^{[4]}$ soient soumis à une surveillance régulière. En revanche, dans la population générale, la recherche des signes cliniques liés aux cancers du pancréas, ainsi que le dosage biologique des enzymes pancréatiques et de l'antigène CA 19-9, n'ont pas la spécificité nécessaire, et sont donc de peu d'utilité préventive. Ils gardent cependant toute leur utilité dans l'algorithme diagnostique chez des patients symptomatiques et suspects. Les difficultés du dépistage des tumeurs du pancréas confinent l'imagerie dans un rôle de seconde ligne où elle doit établir des critères de caractérisation et de résécabilité tumorale. Dans cet article, la stratégie diagnostique tirant le meilleur parti des principales techniques que sont l'échographie, la tomodensitométrie (TDM), l'imagerie par résonance magnétique (IRM) et la tomographie par émission de positrons (TEP) fait l'objet d'une discussion exhaustive. Les principaux effets de l'avènement des techniques en coupes sont la relégation au second plan des techniques diagnostiques ancillaires comme la cholangiopancréatographie rétrograde endoscopique (CPRE) et l'artériographie, ainsi que la diminution du nombre de laparotomies exploratrices.

\section{- Considérations techniques en imagerie du pancréas}

L'échographie, la TDM, l'IRM et la TEP sont les techniques d'imagerie actuellement le plus souvent utilisées pour le diagnostic et la mise au point des tumeurs pancréatiques. Quelques approches consensuelles sur la manière d'acquérir et de traiter les images ont récemment vu le jour, alors que d'autres sont toujours matière à discussion.

\section{Échographie}

L'examen échographique du pancréas se pratique à jeun par voie transabdominale médiane. Cet examen de première ligne et facilement accessible possède l'avantage de se réaliser en temps réel, et d'explorer de manière non irradiante le pancréas, le foie, les voies biliaires et les organes adjacents. Récemment, les techniques d'imagerie harmonique tissulaire, de balayage angulaire multiple et de filtrage adaptatif ont permis d'améliorer le rapport signal sur bruit de l'image échographique sur les transducteurs de basse fréquence utilisés pour l'imagerie du pancréas, ceci sans effet sur la cadence de calcul des images. La technologie harmonique permet également une exploration de meilleure qualité en profondeur en améliorant la visualisation du pancréas et des structures rétropéritonéales.

La faisabilité de l'échographie transabdominale reste tributaire du statut pondéral des patients, de la fenêtre acoustique et de l'opérateur. Afin de pallier ces inconvénients, l'approche endoscopique qui permet de conduire des sondes échographiques radiales ou linéaires de haute résolution directement au contact du pancréas via la lumière gastrique ou duodénale a vu le jour au cours des années 1980. Cet appareillage peut être combiné à une aiguille permettant en temps réel un prélèvement cytologique par aspiration du tissu pathologique. La technologie harmonique a permis le développement et l'utilisation d'agents de contraste échographiques intravasculaires renforçant les fréquences harmoniques et permettant une étude de la perfusion. Ils agissent en augmentant aussi bien l'intensité du signal Doppler couleur que le contraste entre tissus sain et tumoral en mode B-harmonique. Ces agents de contraste peuvent être utilisés aussi bien en échographie transabdominale ${ }^{[5]} \mathrm{qu}^{\prime} \mathrm{en}$ échoendoscopie ${ }^{[6]}$.
Tableau 1.

Délai moyen (secondes) entre le début de l'injection et l'acquisition des différentes phases en tomodensitométrie, en fonction du débit ${ }^{[8]}$.

\begin{tabular}{clllc}
\hline Débit & $\begin{array}{l}\text { Phase } \\
\text { artérielle }\end{array}$ & $\begin{array}{l}\text { Phase } \\
\text { pancréatique }\end{array}$ & $\begin{array}{l}\text { Phase } \\
\text { hépatique }\end{array}$ & $\begin{array}{l}\text { Phase } \\
\text { d'équilibre }\end{array}$ \\
\hline $3 \mathrm{ml} / \mathrm{s}$ & 30 & 50 & 70 & $>120$ \\
$4 \mathrm{ml} / \mathrm{s}$ & 25 & 45 & 65 & $>120$ \\
$5 \mathrm{ml} / \mathrm{s}$ & 20 & 40 & 60 & $>120$ \\
\hline
\end{tabular}

66 Point fort

\section{Échographie}

- Technique largement disponible.

- Réalisation en temps réel.

- Technique non irradiante.

- Possibilité d'utilisation de contraste intraveineux.

- Possibilité d'utilisation endoscopique interventionnelle.

\section{Tomodensitométrie}

La TDM est une technique efficace qui est, de par sa disponibilité, la technique la plus souvent utilisée après l'échographie en cas de suspicion de cancer pancréatique. Sur le plan technique, aucune étude à notre connaissance n'a démontré la supériorité d'une opacification orale au moyen d'un agent de contraste positif par rapport à un agent de contraste négatif dans le bilan d'extension péritonéal des cancers pancréatiques. En pratique, l'administration orale d'un agent de contraste positif altère la qualité des reconstructions vasculaires et peut masquer des calcifications. Afin d'obtenir une meilleure distension gastrointestinale et de limiter le péristaltisme, le décubitus latéral droit et la spasmolyse intraveineuse ont été proposés ${ }^{[7]}$. L'acquisition en contraste spontané est débattue pour deux raisons: d'une part les calcifications qu'elle permettrait de démontrer sont également visibles après contraste intraveineux et, d'autre part, l'analyse de l'évolution des densités tissulaires dans le temps après l'administration intraveineuse d'un agent de contraste iodé permet de différencier un rehaussement d'une zone spontanément hyperdense mais non rehaussée ${ }^{[7]}$. La technique par multidétecteurs restitue les données extraites de la rotation de tubes à rayons $\mathrm{X}$ auxquels fait face une rangée de plusieurs détecteurs. Cette technique, combinée à un accroissement de la vitesse de traitement informatique et des capacités de stockage, a permis de diminuer d'un facteur de près de dix l'épaisseur des coupes et le temps d'acquisition. La TDM permet donc actuellement d'étudier plusieurs «volumes» de voxels isotropiques de près de $1 \mathrm{~mm}^{3}$ acquis en quelques secondes à un temps déterminé par rapport à l'administration intraveineuse d'un agent de contraste iodé. Le Tableau 1 définit les différentes phases en fonction du débit d'injection chez des patients dont la fonction cardiaque n'est pas altérée ${ }^{[8]}$. La vitesse des nouveaux scanners a été mise à profit par certaines équipes pour réaliser une phase artérielle «pure» destinée à préciser le bilan vasculaire, parfois au détriment du nombre de phases. Cette phase artérielle n'a cependant pas démontré sa supériorité par rapport à la phase pancréatique dans le bilan de résécabilité des cancers pancréatiques ${ }^{[8,9]}$. En revanche, elle peut permettre une meilleure détection des tumeurs hypervasculaires ${ }^{[10]}$.

\section{Imagerie par résonance magnétique}

Les examens doivent idéalement être réalisés après spasmolyse intraveineuse. L'opacification digestive peut être positive ou négative en pondération T2, en fonction de l'indication de l'examen. En pratique, des coupes en pondération T2, diffusion et en écho de gradient en pondération T1 en phase et en opposition de 
66 Point fort

\section{Tomodensitométrie}

- Grande résolution spatiale.

- Grande rapidité d'acquisition tridimensionnelle permettant des reconstructions vasculaires.

- Possibilité de détection des calcifications.

- Large champ de vue.

- L'acquisition peut être couplée à celle d'une TEP.

phase sont obtenues avant une étude dynamique pondérée T1 avec saturation du signal de la graisse avant et après administration intraveineuse d'un chélate de gadolinium. Les phases d'acquisition dynamique après contraste intraveineux sont similaires à celles de la TDM. Les propriétés non irradiantes de l'IRM permettent de s'affranchir de la limitation du nombre de phases, rendant possible l'acquisition en routine de la phase artérielle et de la phase pancréatique. Les séquences à forte pondération T2 doivent également être réalisées, car elles permettent d'obtenir une imagerie canalaire sans avoir recours aux agents de contraste à excrétion biliaire. L'usage d'un autre type d'agent de contraste (à base de manganèse), le mangafodipir trisodium, qui possède la propriété de se fixer sur le pancréas et le foie, à l'inverse des tissus tumoraux, s'est avéré supérieur à la TDM pour la délimitation de petites tumeurs pancréatiques et hépatiques ${ }^{[11]}$. Ce type d'agent de contraste n'est cependant pas recommandé en routine dans la littérature.

\section{6}

\section{Point fort}

\section{Imagerie par résonance magnétique}

- Technique non irradiante.

- Excellente résolution temporelle.

- Excellente caractérisation tissulaire.

- Possibilité de réaliser une cholangiopancréatographie.

\section{Tomographie par émission de positrons}

La TEP est une technique d'imagerie fonctionnelle dont le marqueur le plus souvent utilisé en oncologie est le 18-fluorodésoxy-glucose (18-FDG). Cette molécule est un analogue du glucose; elle est activement transférée au sein des cellules exprimant à leur surface un transporteur du glucose de la famille GLUT (GLUT 1 à 5), phosphorylée par une hexokinase (glucose6-phosphatase) en un substrat, le 18-FDG-6-phosphate, qui est ensuite stocké dans les cellules. L'expression du transporteur membranaire est régulée par les besoins du métabolisme, et excessive dans la plupart des tumeurs ${ }^{[12]}$. En pratique, l'acquisition des images se fait une heure après administration intraveineuse du 18-FDG. Cette technique produit des images de contraste extrêmement marqué entre les tissus anormalement hypermétaboliques et les tissus sains. La résolution spatiale des images classiques d'émission est de l'ordre du centimètre. L'acquisition TEP est désormais systématiquement couplée à une étude TDM qui permet de corriger l'atténuation des images en fonction de la densité des tissus et d'augmenter la résolution spatiale de la technique ${ }^{[13]}$ La TEP est une technique complémentaire de la TDM et de l'IRM dans l'évaluation du pancréas, spécialement pour sa capacité à distinguer les cancers de certaines affections non cancéreuses, détecter les métastases ganglionnaires, détecter les métastases occultes à distance, et détecter précocement les récidives chez les patients opérés. Malgré l'impact remarquable de cette technique, elle ne peut pas être utilisée en première ligne pour la détection des cancers pancréatiques en raison de son coût. De plus, quelques tumeurs peuvent être faussement négatives et quelques anomalies non cancéreuses du pancréas s'avérer 18-FDG-avides.

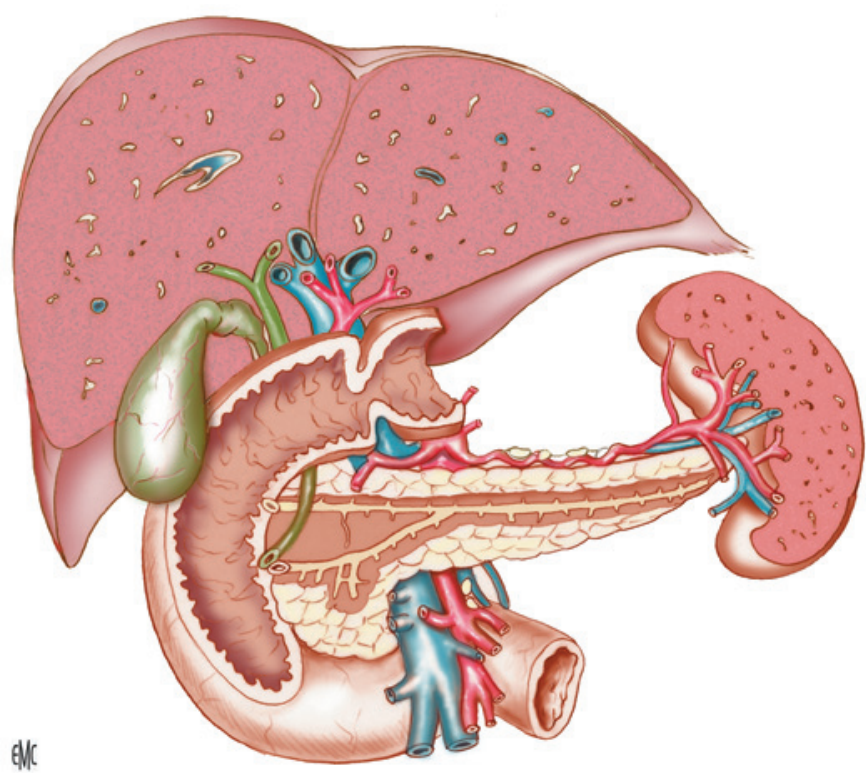

Figure 1. Représentation anatomique schématique du pancréas, de ses canaux de drainage exocrines, des voies biliaires et des organes de voisinage.

Tableau 2.

Intervalles de confiance à $95 \%$ des valeurs diagnostiques des principaux examens d'imagerie dans la détection des tumeurs pancréatiques $[14,15,24]$

\begin{tabular}{lll}
\hline & Sensibilité (\%) & Spécificité (\%) \\
\hline IRM & $84(78-89)$ & $82(67-92)$ \\
TDM & $91(86-94)$ & $85(76-91)$ \\
Échographie & $76(69-82)$ & $75(51-89)$ \\
Échographie avec contraste & $87($ Ind) & 94 (Ind) \\
Échoendoscopie & $77(71-81)$ & $92(86-95)$ \\
TEP & $90(86-93)$ & $76(69-82)$ \\
TEP/TDM & $90(87-93)$ & $76(66-84)$
\end{tabular}

IRM : imagerie par résonance magnétique; TDM : tomodensitométrie; TEP : tomographie par émission de positrons; Ind : indéterminé.

66 Point fort

Tomographie par émission de positrons

- Technique d'imagerie métabolique.

- Bilan locorégional et à distance.

- Acquisition systématique d'une TDM.

- Complémentarité avec I'IRM et la TDM.

\section{Éléments sémiologiques en imagerie pancréatique}

La Figure 1 représente l'anatomie régionale normale du pancréas. Pour toutes les techniques d'imagerie, l'approche sémiologique des tumeurs du pancréas est similaire et regroupe: les anomalies de la trophicité, la dilatation des voies biliaires et des canaux pancréatiques, le changement des propriétés tissulaires et l'envahissement des structures locorégionales. Sur cette base, le Tableau 2 résume les valeurs diagnostiques des principaux examens d'imagerie dans la détection des tumeurs pancréatiques ${ }^{[14,15]}$. 

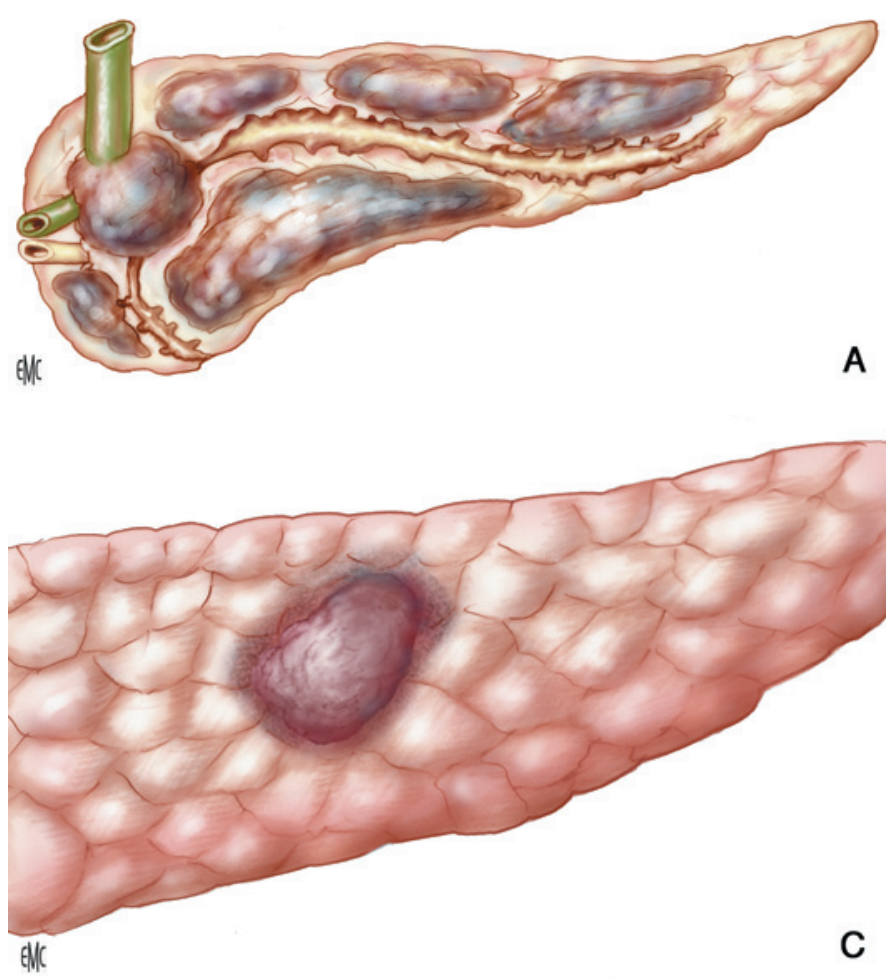

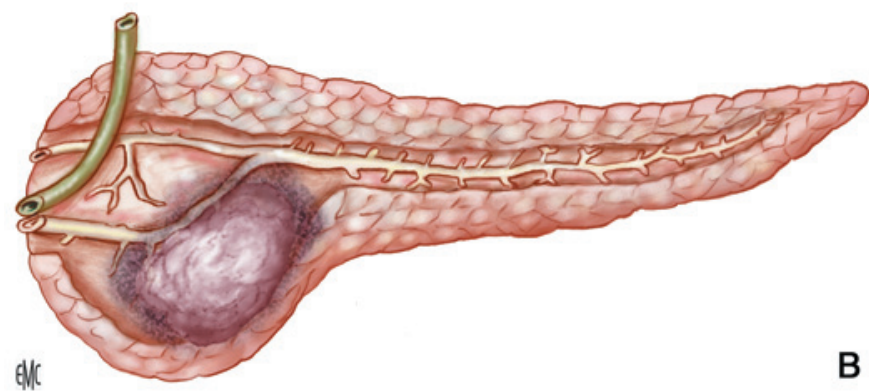

Figure 2. Représentation schématique de l'hypertrophie pancréatique résultant d'un processus tumoral. L'envahissement tumoral de la glande pancréatique peut être diffus (A) ou localisé. L'atteinte localisée se manifeste par une anomalie de contour (B) ou un effacement des lobulations (C).

\section{Anomalies de la trophicité}

L'hypertrophie de la glande pancréatique est souvent retrouvée dans les pathologies tumorales. Elle peut être globale $(5 \%)$ ou focale (95\%) (Fig. 2) ${ }^{[16]}$. En raison des importantes variations individuelles de volume du tissu pancréatique, cette augmentation de taille est estimée de manière subjective par comparaison avec le tissu normal adjacent. L'hypertrophie globale résulte plus souvent d'une pancréatite rétro-obstructive que d'une infiltration tumorale diffuse. Cette hypertrophie peut être difficile à estimer lorsque l'augmentation de volume est discrète et que les autres signes sont absents ${ }^{[16]}$. En cas d'hypertrophie focale, l'atteinte peut parfois être si discrète que seule est démontrée une petite anomalie de contour, ou une disparition focale de l'aspect lobulaire de la glande ou des interstices graisseux du tissu de soutien pancréatique.

\section{Voies biliaires et canaux pancréatiques}

La dilatation d'une structure canalaire doit faire rechercher une tumeur à hauteur de la zone où celle-ci retrouve un calibre normal (zone transitionnelle) ou, à défaut, à son extrémité distale (Fig. 3). La dilatation des voies biliaires et des canaux pancréatiques résulte d'une invasion directe de ceux-ci par la tumeur ou d'une compression. Il s'agit d'un signe objectif, car les canaux secondaires du pancréas et les voies biliaires intrahépatiques sont des structures en principe virtuelles en imagerie conventionnelle. Chez le sujet sain, le diamètre moyen du canal de Wirsung est de $3 \mathrm{~mm}$ et peut varier de 1 à $6 \mathrm{~mm}^{[17]}$. Le cholédoque intrapancréatique doit mesurer moins de $7 \mathrm{~mm}(9 \mathrm{~mm}$ chez les patients après cholécystectomie) et les voies biliaires intrahépatiques moins de $3 \mathrm{~mm}$. Dans les tumeurs de la tête du pancréas, les voies biliaires et les canaux pancréatiques sont dilatés dans $77 \%$ des cas, les voies biliaires seules dans $9 \%$ des cas et les canaux pancréatiques seuls dans $12 \%$ des cas ${ }^{[16]}$ (Fig. 4). L'association de la dilatation des voies biliaires et des canaux pancréatiques est donc fortement suggestive d'un cancer, même si aucun syndrome de masse n'est démontré ${ }^{[18]}$. Cet aspect de dilatation bicanalaire se présente dans environ $5 \%$ des cas et nécessite alors une exploration échoendoscopique ou une CPRE.

\section{Canaux pancréatiques}

Karasawa et al. ${ }^{[19]}$ ont décrit quatre types anatomiques de dilatation du canal pancréatique principal qui sont:

- la dilatation régulière (type 1 ):

- la dilatation régulière en collier de perles (type 2 );

- la dilatation irrégulière (type 3 ) ;

- la dilatation irrégulière avec calculs canalaires (type 4).

Les fréquences respectives de ces dilatations avec un cancer pancréatique sont d'environ $40 \%$ pour les types 1 et 2 , moins de $15 \%$ pour le type 3 et $2 \%$ pour le type 4 . La combinaison d'une dilatation du canal de Wirsung avec une atrophie pancréatique traduit la chronicité de l'obstacle et peut suggérer une tumeur, notamment si le rapport entre la section pancréatique et la section canalaire est inférieur à 0,5 . La visualisation des canalicules pancréatiques secondaires est un signe pathologique, malgré la résolution spatiale sans cesse croissante des nouvelles techniques d'imagerie. Enfin, ces dilatations canalaires et canaliculaires peuvent prendre un aspect kystique qui doit être différencié des pseudokystes et des tumeurs kystiques (Fig. 4).

\section{Voies biliaires}

La dilatation des voies biliaires par invasion tumorale directe survient lorsque le processus pathologique est situé à la tête ou à l'isthme du pancréas. Ce critère n'est cependant pas absolu, car des tumeurs (même de volume important) situées en regard du processus unciné peuvent ne pas entraîner de dilatation des voies biliaires. Dans certains cas, la vésicule et les voies biliaires intrahépatiques peuvent être les seules structures dilatées, notamment lorsque la tumeur s'accompagne d'adénopathies hilaires hépatiques provoquant une compression biliaire.

\section{Changement des propriétés tissulaires}

Le pancréas est un organe contenant du tissu adipeux en quantité variable selon la trophicité glandulaire. La richesse protéique du contenu acinaire fait du pancréas sain un organe spontanément en hypersignal par rapport au foie en pondération T1. Les tumeurs épithéliales pancréatiques se développent sur une matrice mucineuse ou fibreuse le plus souvent hypervascularisée. Au sein de ce tissu se développent des aires de nécrose qui 

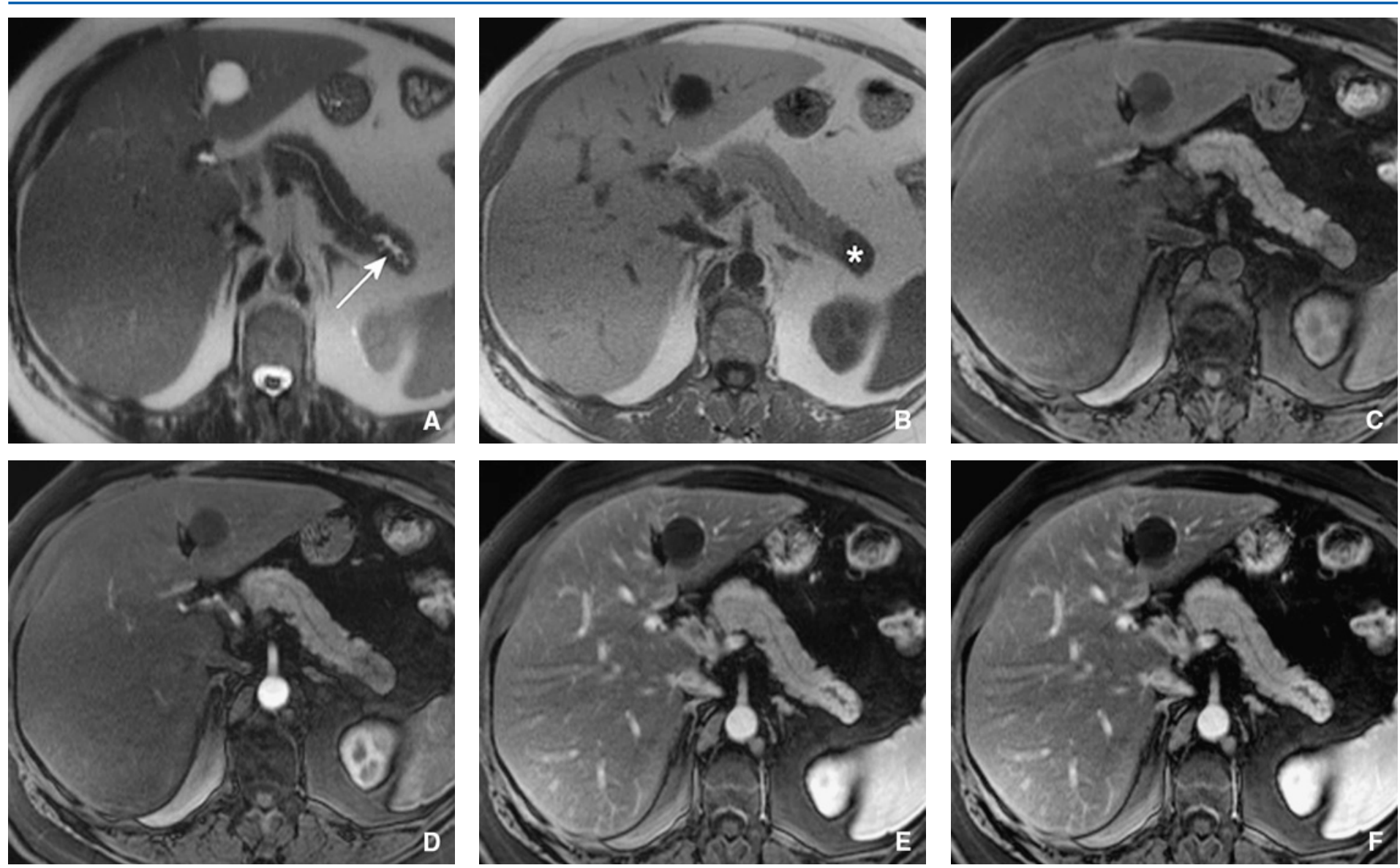

Figure 3. Petit adénocarcinome canalaire du pancréas se manifestant par une dilatation canalaire postobstructive visible en pondération T2 (A) (flèche), un hyposignal en pondération T1 (B) (astérisque) et un rehaussement retardé du pancréas caudal sur l'étude post-contraste dynamique en pondération T1 avec saturation du signal de la graisse (C à F).
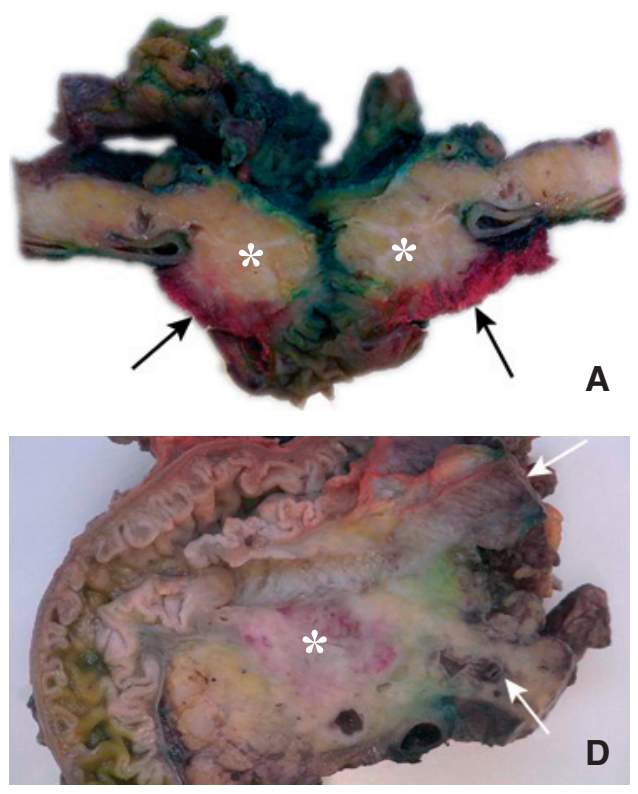
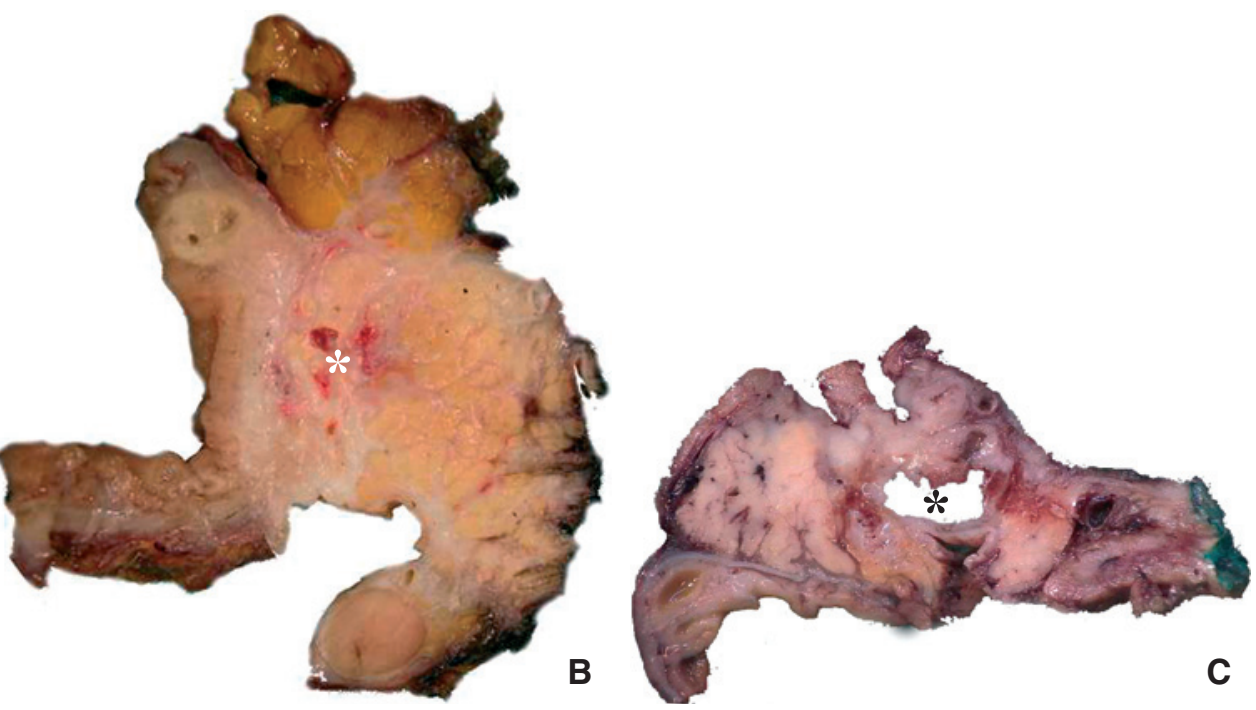

Figure 4. Les adénocarcinomes du pancréas (astérisques) ont sur le plan macroscopique un aspect solide et squirreux (A), parfois hémorragique (B) kystique, mixte (C) ou nécrotique (D). L'envahissement des organes de voisinage est associé à un accolement à ces derniers (A, flèches), tandis que la compression ou l'envahissement des voies biliaires peut être responsable d'une dilatation postobstructive ( $D$, flèches) nécessitant un drainage et la mise en place d'une prothèse dont les mailles sont visibles au sein du relief endoluminal du cholédoque. 
Tableau 3.

Propriétés tissulaires des principaux composants des tumeurs pancréatiques par rapport au tissu sain en imagerie.

\begin{tabular}{|c|c|c|c|c|c|c|}
\hline & \multirow[t]{2}{*}{ TDM $^{\mathrm{a}}$} & \multicolumn{3}{|l|}{ IRM $^{\mathrm{a}}$} & \multirow[t]{2}{*}{ Échographie ${ }^{a}$} & \multirow[t]{2}{*}{ TEP $^{a}$} \\
\hline & & T1 & $\mathrm{T} 2$ & Coefficient de diffusion & & \\
\hline Tissu tumoral & $=/-$ & - & + & - & - & +++ \\
\hline Calcifications & +++ & Variable & $\begin{array}{l}+++ \\
\text { Ombre acoustique }\end{array}$ & $=$ & & \\
\hline Mucus & - & -- & +++ & ++ & $\begin{array}{l}--- \\
\text { Renforcement acoustique }\end{array}$ & - \\
\hline Sang ${ }^{b}$ & ++ & +++ & $+1-$ & ++ & + & - \\
\hline Nécrose & - & - & ++ & ++ & $\begin{array}{l}--- \\
\text { Renforcement acoustique }\end{array}$ & - \\
\hline Pancréatite & - & - & + & - & $+1-$ & + \\
\hline Fibrose & $=$ & $=/-$ & - & $=$ & $=$ & - \\
\hline
\end{tabular}

TDM : tomodensitométrie; IRM : imagerie par résonance magnétique; TEP : tomographie par émission de positrons.

-: structure en contraste négatif par rapport au tissu sain.

$=:$ contraste tissulaire inchangé.

+ à +++: structure de contraste positif d'intensité croissante par rapport au tissu sain.

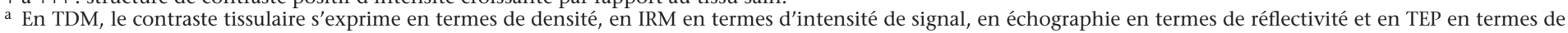
métabolisme.

b Se rapporte à un dépôt hémorragique subaigu.

secondairement peuvent saigner ou se calcifier. Le changement des propriétés tissulaires du pancréas normal est à l'origine de toutes les formes de contraste observables en imagerie classique et en imagerie de diffusion (Tableau 3). Le degré d'hypervascularisation peut être apprécié en TDM ou en IRM de manière dynamique après administration intraveineuse d'agents de contraste extracellulaires. Ces produits de contraste renseignent aussi bien sur la «densité » du réseau vasculaire que sur le volume relatif de la matrice extracellulaire. Ces renseignements sont évidemment d'une grande importance pour la détection et l'évaluation du pronostic des tumeurs qui s'accompagnent d'une angiogenèse dont l'intensité est proportionnelle à l'agressivité ${ }^{[20]}$. La résolution temporelle de la TDM, de l'IRM et de l'échographie permet à certaines équipes d'extraire des paramètres de perfusion tissulaire comme le débit sanguin et le flux sanguin régionaux ${ }^{[20-22]}$. Ces paramètres sont élevés dans les tissus tumoraux à densité vasculaire élevée et leur évolution dans le temps est un paramètre objectif de réponse aux traitements. Ces explorations sont cependant encore à un stade d'évaluation.

\section{Envahissement des structures locorégionales}

Le Tableau 4 détaille la classification TNM des tumeurs pancréatiques. Les tumeurs de stade III et IV ne sont pas éligibles pour une chirurgie (Fig. 5) ${ }^{[23]}$.

\section{6 \\ Point important}

\section{Critères de non-résécabilité des tumeurs pancréa- tiques \\ - Envahissement de la veine porte, de la veine ou de l'artère mésentérique supérieure. \\ - Envahissement du tronc cœliaque, des artères hépa- tiques commune et propre. \\ - Envahissement du plexus cœliaque. \\ - Envahissement de la lame rétroportale. \\ - Envahissement des structures adjacentes comme l'estomac, le côlon, les reins, les surrénales et le rachis. \\ - Métastases hépatiques ou péritonéales.}

La définition précise de ces critères est le champ d'investigation principal de l'imagerie. Le Tableau 5 résume les valeurs diagnostiques des principales techniques d'imagerie dans le bilan global de résécabilité des tumeurs du pancréas. Une méta-analyse rapporte des scores moyens de sensibilité de $81 \%$, $82 \%$ et $83 \%$, et de
Tableau 4.

Classification TNM des tumeurs pancréatiques ${ }^{[23]}$.

\begin{tabular}{|c|c|c|}
\hline \multicolumn{3}{|c|}{ Définitions TNM } \\
\hline \multirow{7}{*}{$\begin{array}{l}\text { Tumeur } \\
\text { (T) }\end{array}$} & TX & Ne peut être défini \\
\hline & T0 & Pas de tumeur démontrée \\
\hline & Tis & Carcinome in situ \\
\hline & $\mathrm{T} 1$ & $\begin{array}{l}\text { Tumeur } \leq 2 \mathrm{~cm} \text { de diamètre, confinée } \\
\text { au pancréas }\end{array}$ \\
\hline & $\mathrm{T} 2$ & $\begin{array}{l}\text { Tumeur }>2 \mathrm{~cm} \text { de diamètre, confinée } \\
\text { au pancréas }\end{array}$ \\
\hline & T3 & $\begin{array}{l}\text { Extension extrapancréatique, sans } \\
\text { atteinte de l'artère mésentérique } \\
\text { supérieure et du tronc coeliaque }\end{array}$ \\
\hline & $\mathrm{T} 4$ & $\begin{array}{l}\text { Atteinte de l'artère mésentérique } \\
\text { supérieure ou du tronc coeliaque }\end{array}$ \\
\hline \multirow{3}{*}{$\begin{array}{l}\text { Ganglions } \\
\text { régionaux }(\mathrm{N})\end{array}$} & NX & Ne peut être défini \\
\hline & No & Pas de ganglion régional envahi \\
\hline & N1 & Ganglion(s) régional(aux) envahi(s) \\
\hline \multirow{3}{*}{$\begin{array}{l}\text { Métastases à } \\
\text { distance (M) }\end{array}$} & MX & Ne peut être défini \\
\hline & MO & Pas de métastase(s) à distance \\
\hline & M1 & Métastase(s) à distance \\
\hline \multirow[t]{9}{*}{ Stade tumoral } & Stade 0 & Tis, N0, M0 \\
\hline & Stade I & \\
\hline & Stade Ia & T1, N0, M0 \\
\hline & Stade Ib & T2, N0, M0 \\
\hline & Stade II & \\
\hline & Stade IIa & T3, N0, M0 \\
\hline & Stade IIb & T1-3, N1, M0 \\
\hline & Stade III & T4, N0-N1, M0 \\
\hline & Stade IV & T1-4, N0-N1, M1 \\
\hline
\end{tabular}

spécificité de $82 \%, 78 \%$ et $63 \%$ pour l'évaluation de la résécabilité des tumeurs pancréatiques, respectivement pour la TDM, l'IRM et l'échographie transabdominale ${ }^{[24]}$. En raison d'une spécificité relativement faible de l'échographie transabdominale et d'un champ d'exploration relativement limité de l'échoendoscopie, la TDM et l'IRM sont actuellement les techniques recommandées pour la réalisation de ce bilan. L'association de la TEP à la TDM offre une alternative séduisante combinant les diagnostics anatomique et métabolique, dont les résultats publiés sont cependant contradictoires d'une série à l'autre ${ }^{[25-27]}$, appelant à des analyses sur de plus larges séries. 

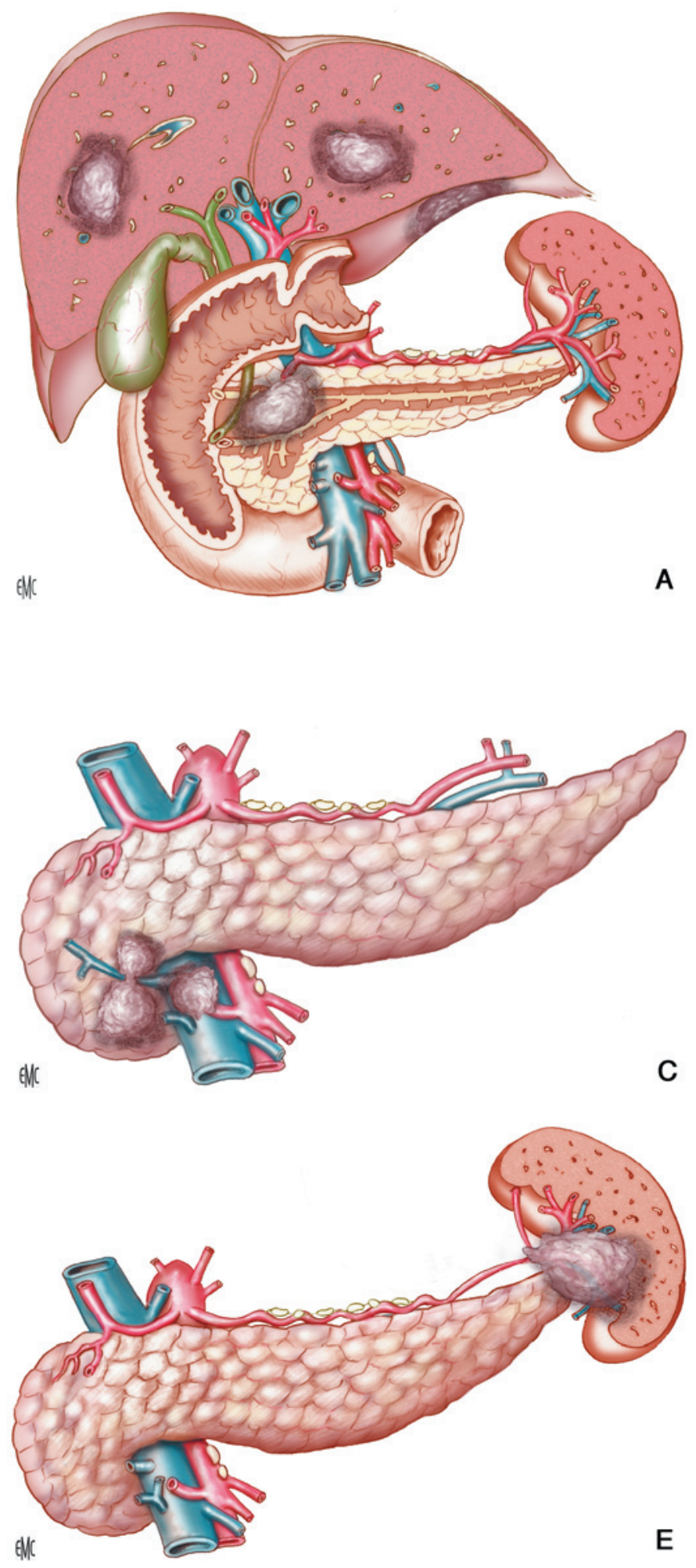

Tableau 5.

Intervalles de confiance à $95 \%$ des valeurs diagnostiques des principaux examens d'imagerie dans le bilan de résécabilité des cancers du pancréas ${ }^{[24,27]}$.

\begin{tabular}{lll}
\hline & Sensibilité (\%) & Spécificité (\%) \\
\hline IRM & $82(69-91)$ & $78(63-87)$ \\
Échographie & $83(68-91)$ & $63(45-79)$ \\
TDM & $81(76-85)$ & $82(77-87)$ \\
TEP & 100 (Ind) & 44 (Ind) \\
TEP/TDM & 96 (Ind) & 82 (Ind) \\
\hline
\end{tabular}

IRM: imagerie par résonance magnétique; TDM: tomodensitométrie; TEP : tomographie par émission de positrons; Ind: indéterminé.
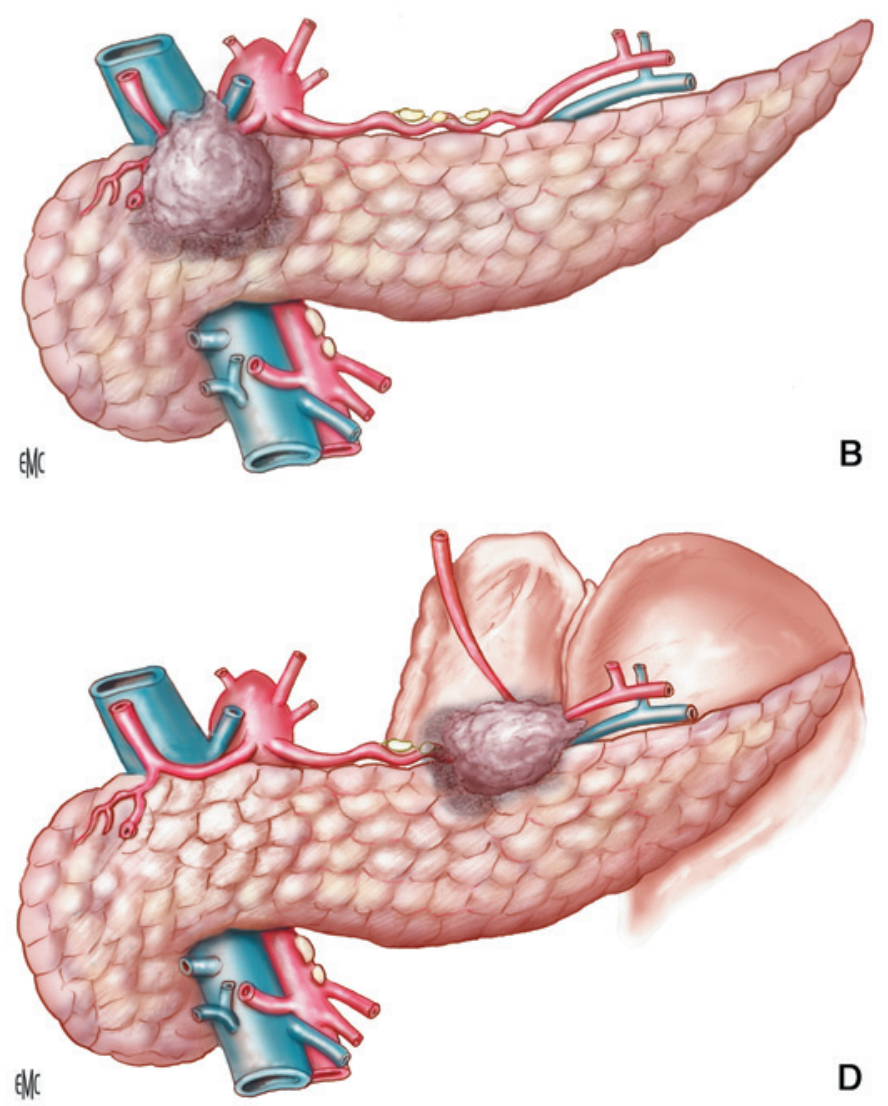

Figure 5. Représentation schématique de tumeurs inopérables du pancréas. Le critère d'inopérabilité peut être la présence d'une carcinomatose hépatique ou péritonéale $(A)$, un envahissement des structures vasculaires régionales telles I'artère hépatique commune (B), la veine mésentérique supérieure (C) et un envahissement des organes de voisinage comme la surrénale (D). L'envahissement splénique isolé (E) n'est pas une contre-indication opératoire.

\section{Envahissement des structures vasculaires}

Le signe le plus spécifique de l'envahissement d'un vaisseau est le rétrécissement de celui-ci; à l'extrême, ce rétrécissement peut conduire à une occlusion. Une distinction doit être réalisée entre l'envahissement veineux et l'envahissement artériel dans la mesure où le premier se solde par une sténose ou une thrombose (Fig. 6), alors que le second se manifeste le plus souvent par un comblement de l'espace périvasculaire par du tissu tumoral, avec effacement du liseré cellulograisseux normalement visible au pourtour du vaisseau (Fig. 7). Pour témoigner d'un envahissement vasculaire, cet effacement doit s'effectuer sur au moins $50 \%$ de la circonférence du vaisseau. Cette valeur-seuil est débattue, puisque plusieurs auteurs utilisent un seuil plus élevé, variant 

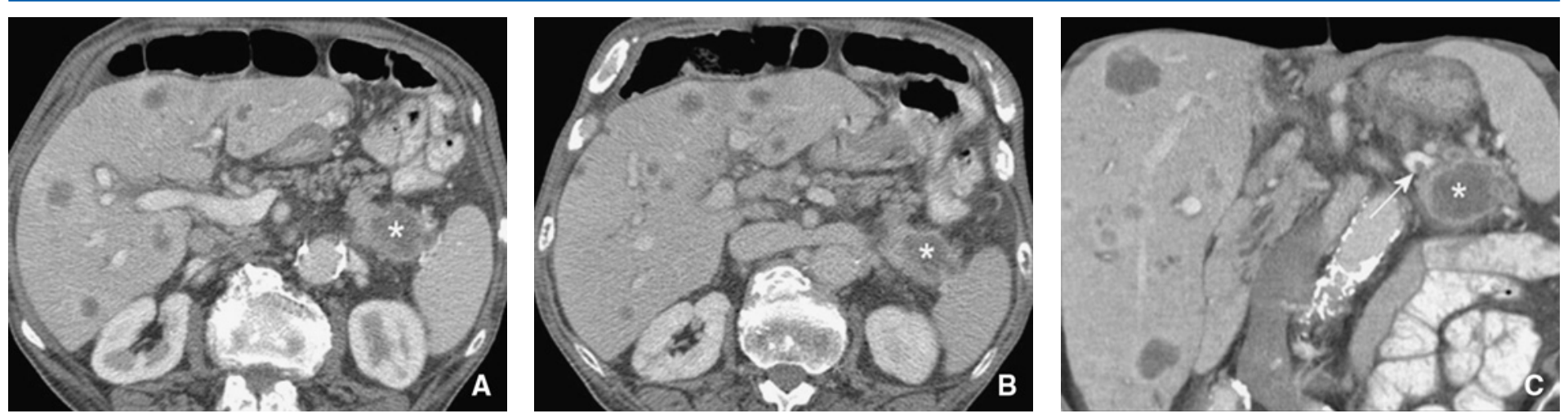

Figure 6. Tomodensitométrie biphasique $(A, B)$ d'un adénocarcinome canalaire de la queue du pancréas (astérisques) démontrant une carcinomatose métastatique hépatique. La reconstruction coronale de la phase hépatique (C) montre une thrombose partielle de la veine splénique (flèche).
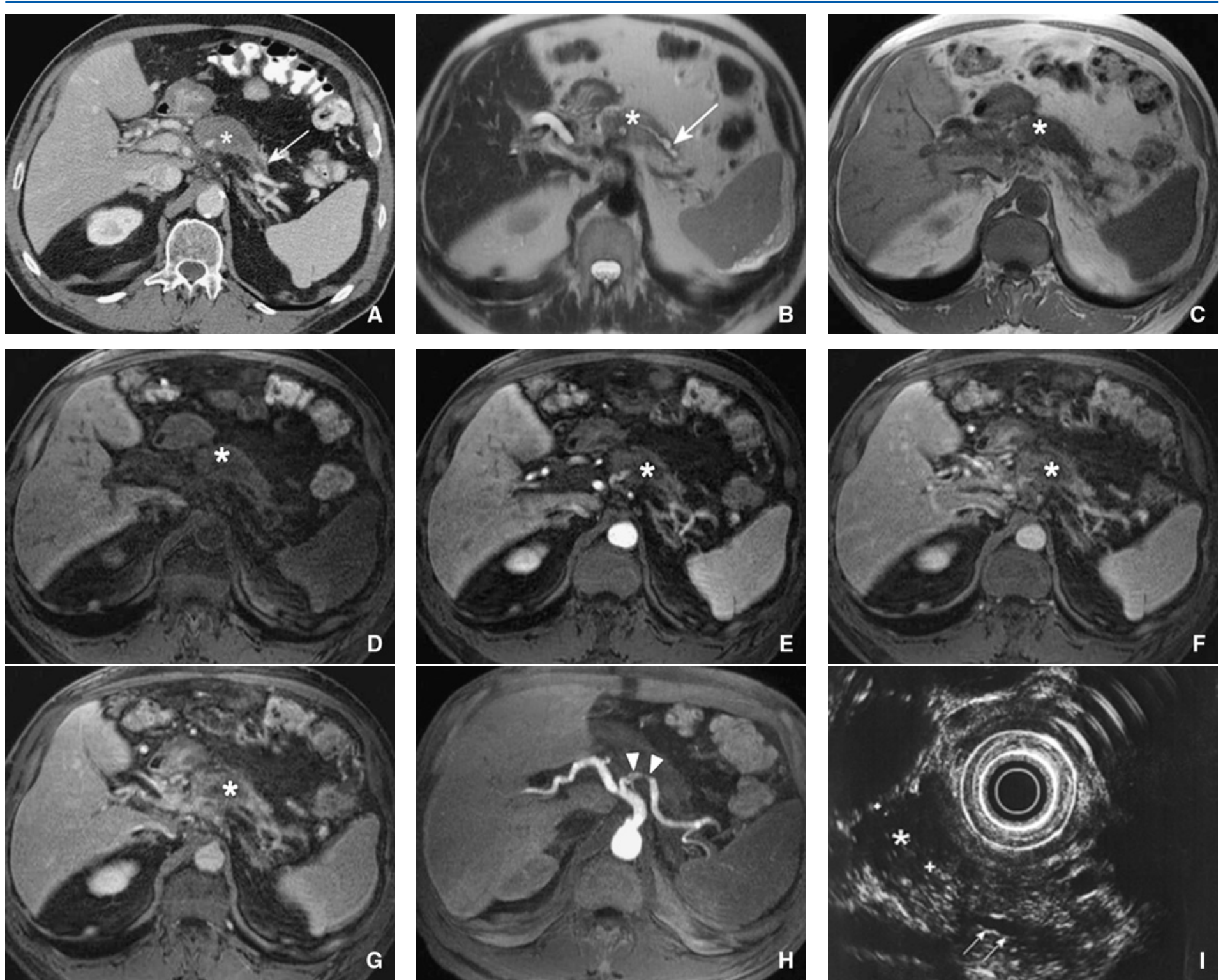

Figure 7. Tomodensitométrie monophasique (A) démontrant une tumeur du corps du pancréas (astérisque) avec dilatation du canal de Wirsung et atrophie du parenchyme pancréatique en amont (flèche). En imagerie par résonance magnétique, la tumeur est en hypersignal en pondération T2 (B) et hyposignal en pondération T1 (C); sa périphérie est progressivement rehaussée par le contraste intraveineux sur l'étude dynamique en pondération T1 avec saturation du signal de la graisse (D à G). Une reconstruction axiale en projection du maximum d'intensité à la phase artérielle $(H)$ permet de démontrer un rétrécissement tumoral de l'artère splénique (têtes de flèches). Le diagnostic d'adénocarcinome canalaire a été posé sur la base d'une biopsie-aspiration pendant l'échoendoscopie (I). 

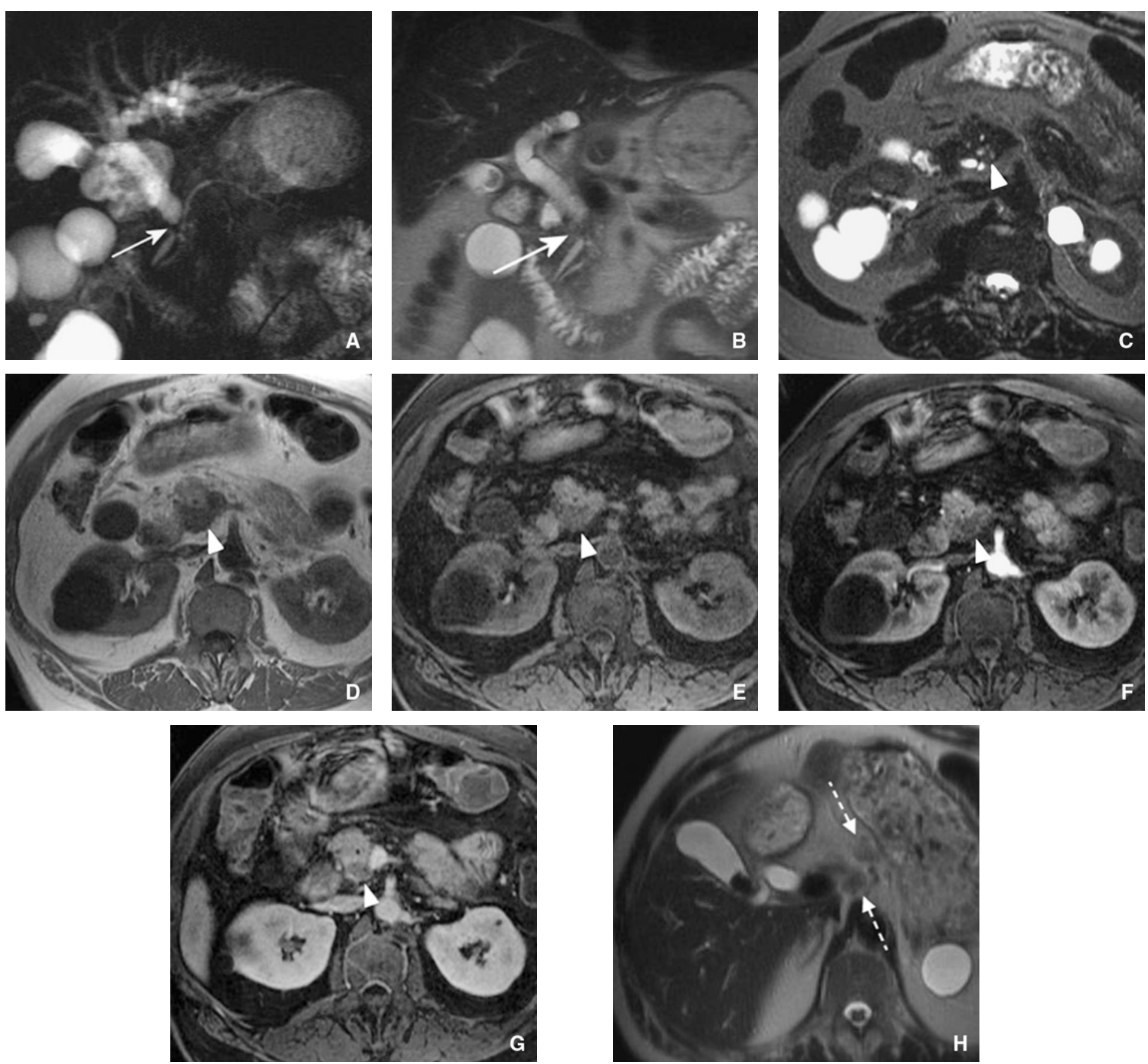

Figure 8. La cholangiopancréatographie en projection (A) et en coupes (B) par imagerie par résonance magnétique démontre une dilatation des voies biliaires intra- et extrahépatiques avec une sténose située dans la portion intrapancréatique du cholédoque (flèche). La dilatation est déterminée par un adénocarcinome canalaire de la tête du pancréas envahissant la lame rétroportale (tête de flèche), au sein duquel on retrouve de fins foyers d'hypersignal en pondération T2 (C). La tumeur est en hyposignal en pondération T1 (D) et présente un rehaussement retardé par rapport au pancréas sur l'étude dynamique post-contraste en pondération $\mathrm{T} 1$ avec saturation du signal de la graisse (E à $\mathrm{G}$ ). Mise en évidence également de ganglions centimétriques gastroduodénaux apparaissant en hyposignal par rapport à la graisse en pondération T2 (H) (flèches en pointillés).

entre $75 \%{ }^{[28]}$ et $90 \%{ }^{[29]}$, sans différences marquées sur les valeurs de sensibilité et de spécificité. Le mésopancréas ou lame rétroportale est une attache conjonctive verticale entre le bord gauche du processus unciné et le bord droit de l'artère mésentérique supérieure, à la face postérieure du carrefour splénomésaraïque et de la veine porte qui constitue un repère anatomique fondamental. En effet, son envahissement, même sans atteinte formelle des structures vasculaires adjacentes, constitue un critère de nonrésécabilité car elle est liée au rétropéritoine (Fig. 8) ${ }^{[30]}$.

Les coupes TDM et IRM acquises de manière dynamique après contraste sont en général nécessaires à cette évaluation. Bien que la valeur ajoutée au diagnostic des reconstructions multiplanaires et en projection du maximum d'intensité à partir des coupes natives soit discutée dans la littérature radiologique, cellesci sont avant tout de nature à faciliter le diagnostic et aider à sa compréhension. Les autres signes d'envahissement des structures vasculaires sont l'irrégularité de la paroi du vaisseau, la visualisation d'un fragment tumoral au sein de la lumière vasculaire et la dilatation des vaisseaux collatéraux ${ }^{[31]}$.

\section{Envahissement lymphatique}

Les espaces péripancréatiques sont riches en relais ganglionnaires qui ont été classifiés en 18 parties différentes devant être examinées dans le cadre du bilan des tumeurs pancréatiques ${ }^{[32]}$. Ces relais peuvent être résumés en relais péripancréatiques (céphaliques, corporéaux et caudaux), lombo-aortiques, cœliaques, gastrohépatiques, gastroépiploïques et mésentériques (proximaux et distaux). Leur accessibilité est variable en fonction de la technique d'imagerie utilisée, et seules la TDM, l'IRM et la TEP 

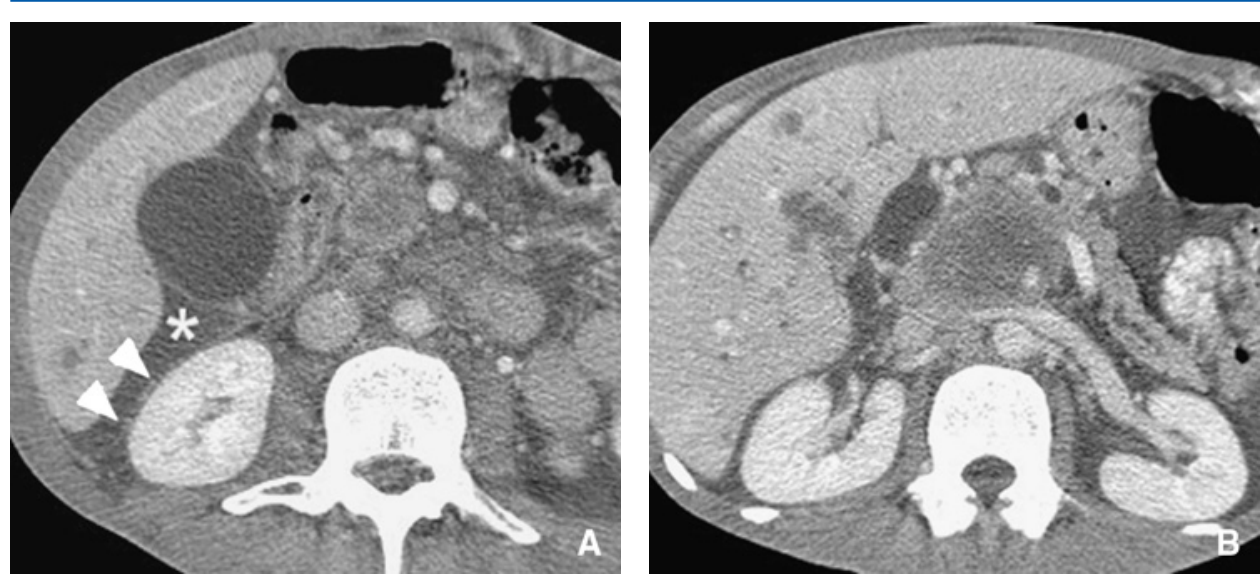

Figure 9. Tomodensitométrie à la phase hépatique d'un adénocarcinome de la tête du pancréas envahissant l'artère mésentérique supérieure et les ganglions péripancréatiques. Présence également de métastases hépatiques et d'une ascite carcinomateuse (astérisque) avec nodules infracentimétriques rehaussés par le contraste intraveineux le long des feuillets péritonéaux périphériques (têtes de flèches) $(A, B)$.

donnent une visualisation globale de ces différents relais. Le signe le plus connu d'envahissement ganglionnaire est l'augmentation de sa taille (Fig. 8). La limite de $10 \mathrm{~mm}$ de largeur pour différencier les ganglions envahis des ganglions sains a été utilisée, avec une sensibilité variant entre 14 et $33 \%$, et une spécificité variant entre 60 et $92 \%{ }^{[33-37]}$. Ces chiffres suggèrent que le critère de taille n'est pas suffisamment sensible pour la détection d'un envahissement ganglionnaire. De plus, la coexistence d'affections inflammatoires locales rend ce critère peu spécifique. $\mathrm{D}^{\prime}$ autres critères d'envahissement tels que la perte de la forme ovoïde normale du ganglion, la non-visualisation de son centre normalement riche en tissu adipeux ou l'agrégation de plusieurs ganglions ont été rapportés comme faiblement prédictifs d'un envahissement tumoral ${ }^{[34]}$. De manière similaire aux autres critères, la détection du métabolisme accru des ganglions en TEP est également décevante. Il est recommandé actuellement que la suspicion d'envahissement tumoral de ganglions péripancréatiques ne soit pas un critère de non-résécabilité, mais permette en revanche d'orienter les prélèvements ganglionnaires lors de l'intervention.

\section{Envahissement des organes adjacents}

L'extension des tumeurs du pancréas aux organes adjacents est un critère de non-résécabilité. Les organes le plus souvent atteints sont l'estomac et le duodénum, ainsi que les organes rétropéritonéaux tels que les reins, les surrénales et le rachis. Les critères retenus sont la perte du liseré graisseux entre la tumeur et l'organe, et l'infiltration de celui-ci par la tumeur. La TDM possède la meilleure résolution spatiale pour ce diagnostic, mais l'IRM doit être préférée lorsqu'il existe une suspicion d'extension au rachis.

\section{Métastases à distance}

La présence de métastases à distance (le plus souvent hépatiques et péritonéales) contre-indique la chirurgie. Sur le plan sémiologique, les métastases hépatiques se présentent comme des lésions focales de propriétés tissulaires différentes de celles du parenchyme hépatique normal. En fonction du type de la tumeur primitive, elles présentent une quantité variable de nécrose centrale. Leurs propriétés spontanées sont donc similaires à celles de la tumeur primitive (Tableau 3). De manière générale, les métastases hépatiques présentent un rehaussement global ou périphérique marqué. Les métastases péritonéales se présentent comme des images additives nodulaires ou parfois linéaires disposées le long des feuillets péritonéaux. Elles peuvent s'accompagner d'ascite en quantité variable. Leur détection en TDM et en IRM requiert le plus souvent une opacification intraveineuse. La spécificité du diagnostic par TDM ou IRM de métastases hépatiques ou péritonéales est comprise entre 95 et $100 \%$. En revanche, pour les deux techniques, la détection des métastases hépatiques et péritonéales infracentimétriques constitue la principale difficulté diagnostique (Fig. 9). En TDM, la sensibilité pour les métastases hépatiques inférieures au centimètre reste inférieure à $80 \%$, même avec la technique multidétecteurs ${ }^{[38]}$. Cette tendance est similaire pour les petites métastases péritonéales. L'IRM semble présenter une meilleure sensibilité pour ce type de lésions dans les séries
Tableau 6.

Types histologiques et fréquence relative des principales tumeurs du pancréas exocrine ${ }^{[23]}$.

\begin{tabular}{ll}
\hline Tumeurs & Pourcentage \\
\hline Tumeurs d'origine canalaire & $\pm 80 \%$ \\
Adénocarcinome canalaire & $6 \%$ \\
Variants de l'adénocarcinome canalaire & \\
Carcinome anaplasique & \\
$\quad$ Non différencié & \\
$\quad$ À grandes cellules pléiomorphes & \\
$\quad$ À cellules géantes de type ostéoclaste & $1 \%$ \\
Adénocarcinome à cellules en bague à chaton & $4 \%$ \\
Carcinome adénosquameux & $1 \%$ \\
Carcinome mixte canalaire et endocrine & $1 \%$ \\
Adénocarcinome microkystique & \\
Tumeur mucineuse kystique & a \\
Tumeur papillaire mucineuse intracanalaire & $1 \%$ \\
Tumeurs d'origine acinaire & $\pm 3 \%$ \\
Adénocarcinome acinaire & \\
Cystadénocarcinome papillaire & $\pm 1 \%$ \\
Tumeurs d'histogenèse incertaine & \\
Pancréatoblastome & \\
Tumeur pseudopapillaire & $<1 \%$ \\
\hline
\end{tabular}

a Appartiennent au spectre morphologique des tumeurs kystiques du pancréas.

ayant spécifiquement adressé cette question ${ }^{[11,39,40]}$. La précision de la TEP combinée à la TDM dans l'évaluation préopératoire des métastases à distance semblerait supérieure à celle des autres techniques d'imagerie ${ }^{[30,41]}$. Cependant, cette combinaison est inférieure à l'IRM dans la détection des petites métastases hépatiques et péritonéales ${ }^{[42]}$, et cette dernière présente une sensibilité limitée par rapport à la laparoscopie. Ceci démontre qu'il reste une indication pour la laparoscopie préopératoire ou périopératoire dans le bilan des cancers pancréatiques ${ }^{[43]}$.

\section{Types histologiques}

Sur le plan histologique, les tumeurs non épithéliales primitives du pancréas telles que les lymphomes et les sarcomes sont dominées en fréquence par les tumeurs d'origine épithéliale qui comprennent les tumeurs exocrines et les tumeurs endocrines. Les tumeurs endocrines ne sont pas traitées dans cet article. Le Tableau 6 liste et indique la fréquence relative des principaux types histologiques des tumeurs du pancréas exocrine discutées dans cet article ${ }^{[44]}$. Le spectre morphologique des tumeurs kystiques du pancréas, comprenant les tumeurs mucineuses kystiques (cystadénomes, cystadénocarcinomes et adénomes macrokystiques) ainsi que les adénomes microkystiques, et les tumeurs papillaires mucineuses intracanalaires, est également exclu de cet article. 


\section{Adénocarcinome canalaire et variants}

\section{Stratégie diagnostique}

L'adénocarcinome canalaire et ses variants représentent près de $90 \%$ de toutes les tumeurs pancréatiques. Le nombre de cas incidents est estimé à 185000 par an, avec un pic situé entre 60 et 70 ans et une proportion de 1,2 à 1,5 hommes pour une femme ${ }^{[45]}$. Son pronostic est sombre et dépend du stade tumoral. La survie moyenne à cinq ans sans résection est inférieure à $5 \%$ et augmente à près de $20 \%$ chez les patients opérés. Il est malheureusement le plus souvent peu symptomatique avant sa découverte à un stade avancé inopérable. Cette figure classique représente près de $80 \%$ des cas ${ }^{[46]}$

\section{Diagnostic clinique et biologique}

Certains facteurs épidémiologiques ont un lien avec les adénocarcinomes canalaires du pancréas ${ }^{[47-52]}$.

\section{6 Point fort}

Facteurs de risque associés aux adénocarcinomes canalaires du pancréas (population-cible potentielle d'un dépistage)

- Histoire d'épisodes de pancréatite idiopathique.

- Syndromes héréditaires (syndrome nævus multiple mélanome, polypose adénomateuse familiale, cancer héréditaire sein/ovaire 1 et 2 , syndrome non-polypose et cancer colique héréditaire, pancréatite héréditaire, syndromes de Peutz-Jeghers, Li-Fraumeni et ataxietélangiectasie).

- Histoire familiale de cancer du pancréas.

- Tabagisme, histoire professionnelle d'exposition aux amines aromatiques.

La relation entre symptômes et localisation tumorale est importante et peut déterminer le pronostic. L'ictère est présent comme symptôme initial chez près de $75 \%$ des patients, et pour des raisons de proximité avec le système biliaire, 90\% des adénocarcinomes canalaires céphaliques sont associés à un ictère, contre $10 \%$ des tumeurs caudales. Par conséquent, l'ictère nu est la présentation clinique la plus fréquente $(52 \%)$ chez les patients avec une tumeur opérable ${ }^{[53]}$, tandis que la douleur seule est présente chez $85 \%$ de patients avec une tumeur avancée. Mannell et al. ont démontré sur une série de patients opérés que la stéatorrhée préopératoire était associée à une survie prolongée, à l'inverse de l'existence d'une douleur préopératoire ${ }^{[54]}$. Cependant, les symptômes sont peu spécifiques et peuvent se rencontrer dans d'autres affections telles que la hernie hiatale et le reflux gastro-œsophagien, la maladie diverticulaire colique et la maladie lithiasique biliaire. La spécificité de ces symptômes pris ensemble ou combinés est d'environ $20 \%{ }^{[55]}$. D'autre part, même présents, les symptômes peuvent être si subtils que les patients les tolèrent aisément, avec pour conséquence un retard important dans le diagnostic ${ }^{[56]}$.

Le diagnostic d'adénocarcinome canalaire du pancréas peut également s'aider d'une élévation des taux sanguins de l'antigène CA 19-9. Pour une valeur-seuil de $37 \mathrm{UI} / \mathrm{ml}$, la sensibilité et la spécificité sont respectivement de 70 et de $87 \%$; lorsque ce seuil est porté à $70 \mathrm{UI} / \mathrm{ml}$, ces valeurs sont 86 et $87 \%{ }^{[57,58]}$. Les faux-positifs sont causés par la maladie lithiasique biliaire, la cholécystite, la cholangite et toutes les formes d'obstruction des voies biliaires.

\section{Aspect macroscopique}

L'adénocarcinome canalaire du pancréas est diffus dans $5 \%$ des cas, céphalique dans $60 \%$ des cas et corporéocaudal dans les cas restants. Sur le plan pathologique, l'apparence classique est celle d'une tumeur solide squirreuse et infiltrante, presque toujours accompagnée de phénomènes inflammatoires au sein du pancréas adjacent. La nécrose centrale et la dégénérescence mucineuse ne sont pas rares et peuvent donner à la tumeur un aspect
66 Point fort

Symptômes et signes cliniques associés aux adénocarcinomes canalaires du pancréas

- Ictère obstructif.

- Perte de poids inexpliquée.

- Douleur abdominale inexpliquée, irradiant vers le dos.

- Dyspepsie.

- Diabète sucré d'apparition brutale.

- Stéatorrhée inexpliquée.

- Cachexie.

- Vésicule palpable (signe de Courvoisier).

- Adénopathie supraclaviculaire palpable.

- Masse périombilicale.

- Nodule palpable dans l'espace rectovaginal ou dans l'espace rectovésical.

kystique (Fig. 4). Ces remaniements chroniques peuvent également se compliquer d'hémorragies et de calcifications intratumorales (Tableau 3). Les tumeurs anaplasiques sont en général extrêmement larges et nécrotiques, s'accompagnant de volumineux ganglions d'aspect similaire; cette apparence est dite sarcomatoïde.

\section{Diagnostic par l'imagerie}

L'échographie transabdominale est la technique d'imagerie vers laquelle sont adressés en première intention les patients suspects d'avoir une masse abdominale. Cette technique est peu coûteuse, rapidement disponible et non irradiante. Elle permet avec une grande fiabilité de diagnostiquer d'autres affections, telles que la maladie lithiasique biliaire et ses complications. Dans des mains expertes, elle identifie aisément une obstruction des voies biliaires extrahépatiques, tout en définissant son niveau. Cette technique est malheureusement dépendante de l'expérience, et les sensibilités varient de moins de $50 \%$ à plus de $90 \%{ }^{[59,60]}$. De plus, même pour les examinateurs les plus expérimentés, un examen échographique complet du pancréas peut être techniquement irréalisable chez 15 à $20 \%$ des patients, pour des raisons qui incluent l'interposition de gaz, des remaniements post-chirurgicaux et l'obésité.

Par conséquent, un examen échographique négatif ne peut exclure une tumeur du pancréas.

L'efficacité et la disponibilité de la TDM en font la pierre angulaire de la détection des adénocarcinomes canalaires chez les sujets avec une histoire clinique évocatrice. De plus, la largeur du champ d'exploration permet de réaliser simultanément un pronostic de résécabilité des tumeurs. La sensibilité de la TDM multicoupes biphasique est supérieure à $90 \%$ pour toutes les tumeurs et avoisine $100 \%$ pour les tumeurs de plus de $2 \mathrm{~cm}^{[8]}$. La complémentarité entre l'IRM et la TDM n'a pas été établie et, le plus souvent, une technique doit être préférée à l'autre. Cette préférence dépend de l'accessibilité de l'appareillage, de l'expertise du centre et du niveau de collaboration du patient. Sans être irradiante, l'IRM partage actuellement les qualités diagnostiques de la TDM, et peut même dans certains cas s'avérer supérieure pour identifier la lésion, grâce à une meilleure caractérisation tissulaire. Elle présente cependant par rapport à la TDM les désavantages d'être plus onéreuse et moins souvent disponible. Ces propriétés tendent à suggérer, dans une simulation mathématique de 2015, un bénéfice du screening par IRM chez des sujets asymptomatiques avec des prédispositions génétiques ${ }^{[61]}$. Dans l'attente d'une confirmation de cette simulation par des études de cohorte, le dépistage du cancer du pancréas par des examens d'imagerie n'est pas recommandé chez des sujets asymptomatiques.

L'échoendoscopie partage avec l'approche transabdominale le caractère opérateur-dépendant et ne peut être utilisée en pratique pour le bilan de résécabilité en raison de son champ d'exploration limité. Comparée à la TDM et à l'IRM, elle a une sensibilité similaire, voire plus élevée pour les tumeurs de moins de $2 \mathrm{~cm}$. 


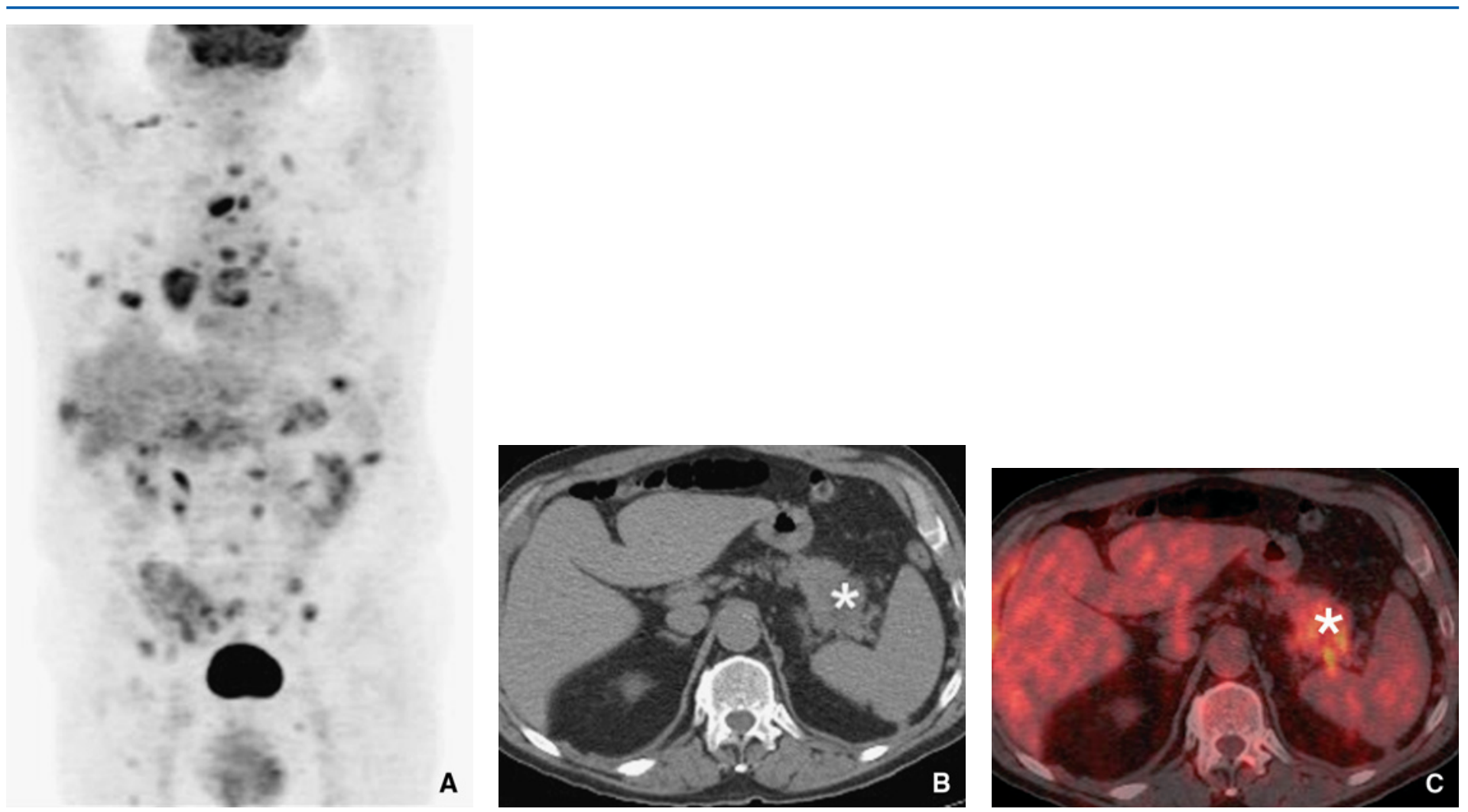

Figure 10. Tomographie à émission de positrons (TEP) en projection du maximum d'intensité (A) chez un patient porteur d'une tumeur bronchique du lobe inférieur droit en dissémination diffuse. Les coupes tomodensitométriques (TDM) en contraste spontané (B) et la fusion TDM-TEP (C) démontrent une masse hypermétabolique de la queue du pancréas (astérisque), représentant une métastase intrapancréatique.

Néanmoins, des valeurs de spécificité de $53 \%$ ont été rapportées ${ }^{[62]}$. Cette faible spécificité est cependant compensée par la possibilité qu'offre cette technique de réaliser une aspiration à l'aiguille fine. Cet examen doit donc être réservé à des patients à deux extrémités sur le plan clinique: ceux pour lesquels, malgré une importante suspicion clinique et radiologique, aucune tumeur n'a formellement pu être démontrée et chez les patients ayant bénéficié d'une TDM dont les résultats sont formels quant à la présence d'une tumeur inopérable, afin de préciser le diagnostic pathologique.

La position de la TEP dans la stratégie diagnostique des adénocarcinomes canalaires du pancréas n'est pas encore clairement définie. Cette technique est souveraine pour l'exploration du corps entier et peut permettre de mettre en évidence des métastases extra-abdominales, des cancers synchrones ou des lésions pancréatiques dans le cadre du bilan de tumeurs situées à distance (Fig. 10). La sensibilité de la TEP est limitée par la taille des lésions et sa spécificité par les réactions inflammatoires chroniques qui simulent l'adénocarcinome canalaire ${ }^{[63]}$. La TEP ne permet pas de distinguer les structures vasculaires et ne peut donc être utilisée pour le pronostic de résécabilité. De plus, la technique est onéreuse. En revanche, sa complémentarité avec la TDM est excellente, se traduisant dans les faits par l'usage quasiment généralisé d'une exploration hybride unique.

\section{Diagnostic pathologique}

L'échoendoscopie a réduit de manière significative le recours à la biopsie percutanée ainsi que le risque d'essaimage péritonéal qui s'y associe. La nécessité d'obtenir un diagnostic histologique lorsque l'histoire de l'affection, les symptômes et les anomalies en imagerie suggèrent un adénocarcinome canalaire du pancréas est sujette à débat, puisque ce diagnostic se vérifie plus de neuf fois sur dix ${ }^{[64]}$. La ponction-biopsie percutanée des adénocarcinomes du pancréas ne s'indique plus que dans les cas où elle peut être réalisée par voie extrapéritonéale chez des patients réputés inopérables pour lesquels le diagnostic de nature est douteux. De manière générale, le diagnostic définitif d'adénocarcinome canalaire est posé sur la pièce de résection. En revanche, lorsque les différentes techniques ne sont pas parvenues à établir le pronostic de résécabilité, le diagnostic est obtenu au moment de la laparoscopie exploratrice qui précise le bilan vasculaire et surtout détermine la présence ou l'absence de métastases hépatiques ou péritonéales ${ }^{[65]}$. Sur le plan pratique, Katz et al. intègrent les progrès de l'imagerie en coupes et préconisent comme indications de la laparoscopie exploratrice les tumeurs extrêmement larges, des anomalies TDM suggérant sans certitude des métastases et une forte suspicion clinique de cancer du pancréas, comme par exemple une importante élévation du taux sanguin de CA 19-9.

\section{Traitement curatif}

Le détail des procédures chirurgicales du pancréas est décrit par ailleurs. Quelques points restent cependant d'intérêt dans la discussion du présent article. Le traitement classique des adénocarcinomes canalaires du pancréas est la duodénopancréatectomie céphalique, décrite par Whipple en 1942. Au cours de cette procédure, de 40 à $50 \%$ du sac gastrique (antre), la vésicule, le cholédoque distal, la tête du pancréas, le duodénum et le jéjunum proximal sont réséqués en bloc. La restitution de la continuité des structures canalaires requiert alors une pancréaticojéjunostomie, une hépatojéjunostomie et une gastrojéjunostomie. Pour des équipes expérimentées, la mortalité opératoire de cette procédure est inférieure à $2 \%{ }^{[64]}$. La morbidité de la procédure est liée à la pancréaticojéjunostomie qui, pour des raisons microcirculatoires, peut être le siège d'un lâchage de suture. Plus à distance, une perte de poids et des déficits nutritionnels importants sont liés à la gastrectomie partielle. La préservation du pylore, qui est largement pratiquée depuis les années 1970, ne s'accompagne pas d'effet péjoratif sur la survie à cinq ans, mais reste malheureusement également sans effet prouvé sur les troubles nutritionnels et pondéraux post-chirurgicaux.

À l'extrémité opposée de la procédure de Whipple, la splénopancréatectomie caudale s'adresse aux tumeurs de la queue du pancréas. Entre ces deux options se trouve la pancréatectomie complète (ou régionale) qui enlève en bloc la totalité du pancréas, ainsi que l'artère mésentérique supérieure, le tronc cœliaque, la veine porte et la base du mésocôlon transverse. Cette opération a été progressivement abandonnée en raison d'une mortalité opératoire et d'une morbidité (diabète instable) plus élevées que pour 
les autres techniques, pour un effet non démontré sur la survie à cinq ans. Elle se pratique néanmoins encore pour les patients dits «marginalement» opérables chez lesquels il existe un envahissement vasculaire, mais au versant veineux portal ou mésentérique. Les résultats à large échelle de la procédure dans cette indication sont à venir.

\section{Traitement palliatif}

Un certain nombre de gestes chirurgicaux sont destinés à améliorer la qualité de vie chez des patients inopérables. Ces gestes sont destinés à soulager la douleur, ainsi que les symptômes de l'ictère obstructif et de l'obstruction intestinale (duodénale). Ces procédures incluent une anastomose biliodigestive par cholécystojéjunostomie ou cholédocojéjunostomie pour l'ictère obstructif, une gastrojéjunostomie pour l'obstruction duodénale et une neurolyse splanchnique à l'éthanol pour la douleur. Bien que ces procédures soient de faible mortalité, elles peuvent être remplacées par des techniques moins invasives.

La douleur est un symptôme important et parfois insupportable, et peut être difficile à maîtriser par les traitements analgésiques classiques. Elle résulte de l'envahissement tumoral des plexus splanchniques. Le principe du traitement percutané est de guider sous contrôle TDM une aiguille fine jusqu'au plexus cœliaque et d'y injecter une solution de $95 \%$ d'éthanol. Avant cette alcoolisation, le positionnement correct de l'extrémité de l'aiguille doit être vérifié par l'injection de produit de contraste iodé. Celui-ci doit se déposer en position rétropéritonéale, para-aortique et extravasculaire. Cette procédure est presque sans complication, avec des risques théoriques d'hémorragie, d'infection ou de perforation de structures digestives. Dans des études randomisées portant sur des patients non opérables, la neurolyse s'est montrée supérieure au placebo et au traitement pharmacologique classique ${ }^{[66]}$ pour l'analgésie et la survie.

La décompression du système biliaire peut être réalisée par la mise en place de prothèses expansibles. La technique percutanée consiste à ponctionner, décomprimer et opacifier les voies biliaires intrahépatiques, à les cathétériser de manière antégrade avec un guide souple jusqu'au duodénum, puis à larguer une prothèse biliaire de calibre et de longueur variables en fonction des caractéristiques de l'obstacle. Le diamètre interne minimal de la prothèse est de 6 à $8 \mathrm{~mm}$, afin de protéger des risques d'angiocholite et de récidive. Dans un certain nombre de cas, le franchissement de la sténose doit être différé de 48 heures (avec poursuite du drainage externe); il ne peut définitivement être obtenu que dans $10 \%$ des cas, obligeant la procédure à se limiter à un drainage externe. Les complications de cette procédure sont essentiellement infectieuses ou relatives à l'accès percutané, incluant la péritonite biliaire, l'hémopéritoine, l'hémothorax, le pneumothorax, la fistule portobiliaire et la fistule pleurobiliaire. La technique endoscopique requiert un cathétérisme rétrograde des voies biliaires par un guide métallique, avec mise en place d'une prothèse. La faisabilité immédiate de cette procédure (81\%) est supérieure à celle de la technique percutanée $(61 \%)^{[67]}$, mais elle n'offre pas la possibilité de recours à un drainage de décompression en cas d'échec. Comme pour l'accès percutané, les complications sont principalement infectieuses. Les hémorragies et perforations digestives ou biliaires peuvent également survenir, mais avec un taux de complications sensiblement plus faible que pour la technique percutanée dans l'étude randomisée de Speer et al. ${ }^{[67]}$. Les deux techniques partagent également comme complication à moyen terme la réobstruction du système biliaire. Celle-ci peut être due à la progression du cancer, à la migration de la prothèse ou à la prolifération biliaire à l'intérieur de celleci. Ces complications sont plus fréquentes pour des prothèses souples que pour des prothèses métalliques. Il est conseillé, pour les patients porteurs de prothèses en plastique, un remplacement tous les trois mois afin de ne pas s'exposer à cette complication [68] Les patients atteints d'un cancer du pancréas se présentent rarement à la phase initiale avec une obstruction duodénale, mais 15 à $20 \%$ de ceux qui ne sont pas opérés en développent les symptômes dans le décours de leur maladie ${ }^{[69]}$. L'usage de prothèses métalliques d'insertion endoscopique a été rapporté avec succès dans cette indication.
La chimiothérapie et la radiothérapie font actuellement partie de l'arsenal palliatif. Elles s'adressent à des patients inopérables ou dans les essais néoadjuvants de réduction du volume tumoral. Ces traitements extrêmement agressifs ne doivent être utilisés que chez des patients dont l'état physique et la tolérance sont supposés bons. Les principales options incluent une combinaison de radiothérapie et de monochimiothérapie au 5-fluorouracile, ou une monothérapie à la gemcitabine. Le gain en survie de la radiochimiothérapie dans les cancers avancés du pancréas est faible. Des techniques d'ablation non chirurgicale telles que l'ablation par ultrasons en haute fréquence, la radiofréquence, l'électroporation irréversible, la thérapie photodynamique, la radiothérapie stéréotaxique, la radiothérapie et la cryochirurgie à l'iode 125 ont été proposées avec un allongement moyen de la survie plus important ${ }^{[70]}$. L'évaluation sur de plus grandes séries des effets bénéfiques et secondaires de ces différents protocoles de traitements palliatifs reste nécessaire.

\section{Suivi}

Un suivi postopératoire régulier doit être effectué, incluant tous les trois mois pendant les deux premières années et tous les six mois au-delà un examen clinique, le dosage du CA 19-9 et une étude en imagerie. La récidive postopératoire est causée par des cellules tumorales laissées dans le rétropéritoine ou le péritoine pendant l'opération, ou par l'existence méconnue d'une tumeur de stade IV. En suivant régulièrement par échographie, TDM et dosage des marqueurs tumoraux 24 patients ayant subi avec succès une opération de Whipple, Kloppel et Maillet ont observé 20 récidives dans les huit premiers mois, sous la forme d'une infiltration rétropéritonéale chez 18 patients et d'une adénopathie rétropéritonéale chez deux patients. Chez les 20 patients ayant récidivé, la récidive était démontrée par toutes les techniques de suivi dans $50 \%$ des cas, par le suivi en imagerie uniquement dans $25 \%$ des cas et par une élévation des marqueurs tumoraux d'abord dans $25 \%$ des cas. Actuellement, l'examen recommandé dans le suivi est la TDM. Cette exploration requiert une connaissance du montage chirurgical et de ses apparences en coupes, et son degré de performance est négativement affecté par le collapsus et la non-opacification de l'anse afférente. Stumpp et al. ont proposé l'usage de produits de contraste à excrétion biliaire afin de contourner cet écueil technique ${ }^{[71]}$. À défaut, l'IRM peut être réalisée dans les centres qui en possèdent l'expertise. En cas d'élévation du CA 19-9, la TEP est une technique à considérer, en particulier lorsque la TDM ou l'IRM restent négatives ou équivoques ${ }^{[72-74]}$.

\section{Algorithme décisionnel}

Dans la littérature, plusieurs algorithmes décisionnels sont proposés pour le diagnostic et le suivi des adénocarcinomes canalaires du pancréas. La Figure 11 présente un algorithme tiré de la confrontation entre les données de la littérature et l'expérience personnelle.

\section{Autres tumeurs du pancréas exocrine}

Les autres tumeurs du pancréas exocrine discutées ici incluent les tumeurs acinaires et les tumeurs d'histogenèse incertaine incluant la tumeur pseudopapillaire et le pancréatoblastome.

\section{Adénocarcinome acinaire}

Les signes cliniques de l'adénocarcinome acinaire sont similaires à ceux des autres tumeurs pancréatiques, mais cette tumeur peut sécréter en quantité anormale les enzymes pancréatiques exocrines. Cette élévation des taux sériques d'enzymes lipolytiques peut, dans des cas extrêmes, être responsable d'une dissémination de foyers de lipolyse systémique comme dans des épisodes de pancréatite aiguë sévère. Les signes cliniques résultant incluent des nécroses graisseuses sous-cutanées, des arthralgies et des lésions osseuses lytiques. Ce syndrome radioclinique est relativement spécifique de cette tumeur ${ }^{[75]}$. Le diagnostic pathologique est basé sur les résultats de l'étude immuno-histo-chimique positive pour les marqueurs membranaires acinaires et de la 


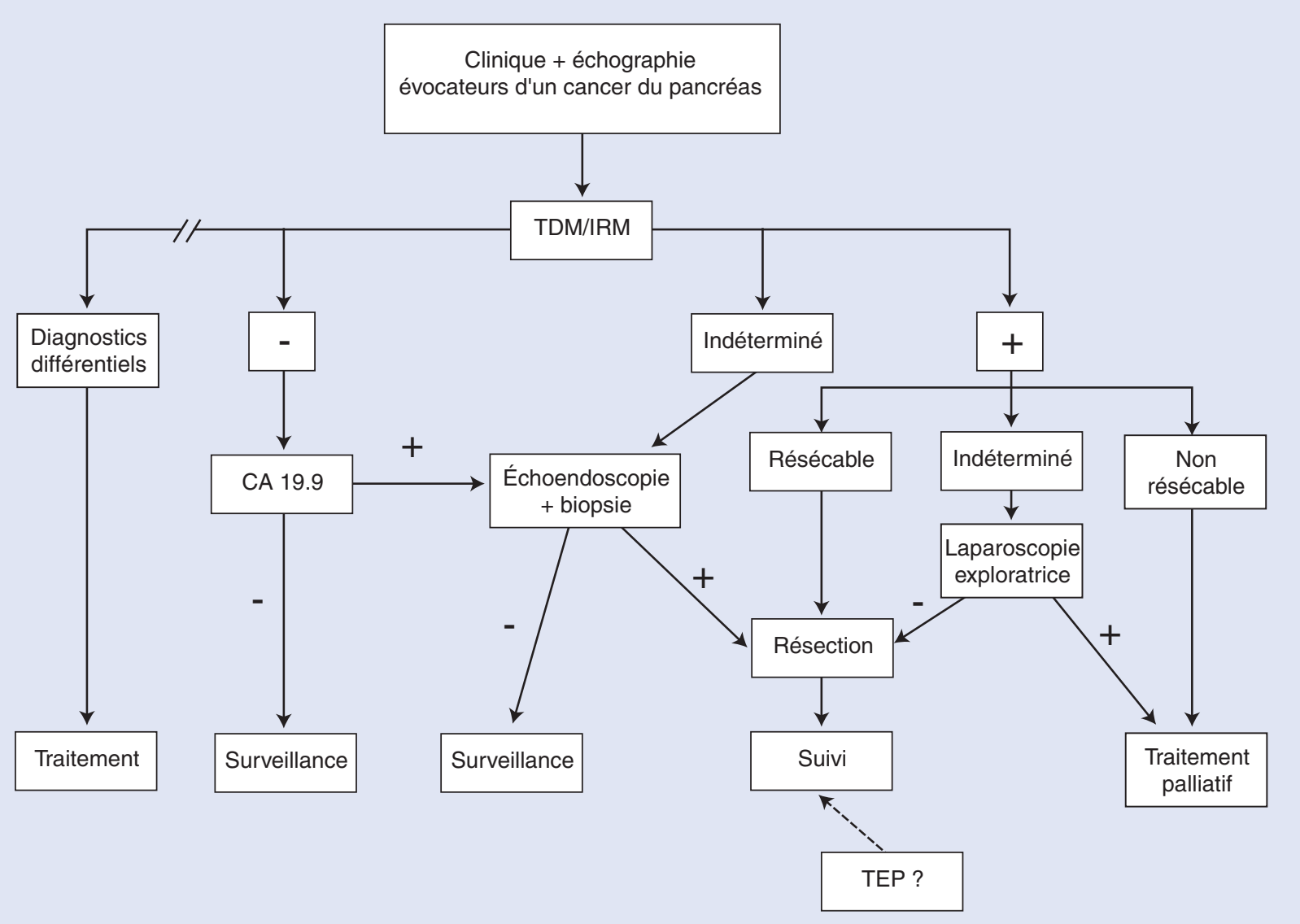

Figure 11. Arbre décisionnel dans le diagnostic et le suivi des tumeurs pancréatiques. TDM : tomodensitométrie; IRM : imagerie par résonance magnétique ; TEP : tomographie à émission de positrons.

microscopie électronique positive pour de nombreux granules cytoplasmiques et un abondant réticulum endoplasmique. Sur le plan radiologique, Chiou et al. ont étudié dix cas et observé une tumeur bien délimitée chez huit patients, parmi lesquels six présentaient une capsule fortement vascularisée. Huit patients présentaient également de la nécrose en quantité variable et cinq présentaient des calcifications au centre de la tumeur. Aucune des tumeurs ne présentait de foyer hémorragique ${ }^{[76]}$. Tatli et al. ont retrouvé des caractéristiques similaires dans leur série ${ }^{[7]}$. Dans une des séries, l'envahissement vasculaire était présent chez la moitié des patients ${ }^{[76]}$ et, dans une autre, un envahissement du duodénum observé sur quatre des cinq masses situées à la tête du pancréas ${ }^{[77]}$. Cette tumeur étant découverte large et exopancréatique, parfois multiple dans le pancréas, son pronostic est équivalent à celui de l'adénocarcinome canalaire, à la différence qu'elle semble cependant davantage radiosensible ${ }^{[78]}$.

\section{Tumeur pseudopapillaire}

Les tumeurs pseudopapillaires sont des tumeurs d'histogenèse incertaine qui sont classiquement découvertes chez la femme jeune. Leur pronostic est excellent comparé à celui des adénocarcinomes classiques du pancréas. Sur le plan pathologique, ces tumeurs sont typiquement larges et encapsulées, contenant des degrés variables de composants solides, kystiques et surtout hémorragiques. Bien que rares, ces tumeurs doivent être diagnostiquées chaque fois que possible. Les données pathologiques offrent la possibilité de s'approcher du diagnostic en imagerie, car les hémorragies intratumorales ne sont pas fréquentes dans les autres types histologiques ${ }^{[79]}$. Leur aspect échographique n'est pas corrélé avec l'apparence histopathologique. En revanche, elles apparaissent hétérogènes en TDM, avec des foyers hyperdenses non calcifiés, et présentent des aires d'hypersignal spontané en pondération T1 en IRM ${ }^{[80]}$.

\section{Pancréatoblastome}

Le pancréatoblastome est une autre tumeur rare du pancréas, d'histogenèse incertaine, touchant principalement les enfants d'âge compris entre 1 et 8 ans, et exceptionnellement les nouveau-nés et les adultes. Bien que certaines formes congénitales aient été décrites en association avec le syndrome de Beckwith-Wiedemann, les pancréatoblastomes sont essentiellement sporadiques et d'étiologie inconnue. Cette tumeur est classiquement constituée d'un mélange d'éléments tumoraux primitifs acinaires, canalaires et endocrines, évoquant une réminiscence de pancréas fotal peu différencié, comme à la septième semaine d'âge gestationnel ${ }^{[81]}$. Plusieurs marqueurs tumoraux tels que le CEA, le CA 19-9 et l'alphafotoprotéine peuvent être élevés ${ }^{[82]}$. Au plan macroscopique, le pancréatoblastome est une tumeur au moins partiellement encapsulée, de consistance variable et hétérogène. Elle peut présenter des aires kystiques et des calcifications macroscopiques; les anomalies en imagerie TDM et IRM reproduisent cette hétérogénéité ${ }^{[81,83]}$. L'envahissement des structures vasculaires n'est pas exceptionnel, mais la survie des patients opérables est meilleure que pour les adénocarcinomes ${ }^{[84]}$.

\section{Diagnostic différentiel}

Le spectre morphologique des tumeurs kystiques du pancréas inclut des lésions congénitales (kystes), inflammatoires (pseudokystes et abcès) et tumorales (adénomes microkystiques, tumeurs mucineuses kystiques, tumeurs mucineuses papillaires intracanalaires et tumeurs endocrines). Ces tumeurs sont décrites par ailleurs, mais peuvent être difficiles à distinguer des tumeurs hétérogènes partiellement kystiques du pancréas exocrine, comme les formes fortement nécrotiques de l'adénocarcinome canalaire, le pancréatoblastome et la tumeur pseudopapillaire ${ }^{[85-87]}$. Le diagnostic différentiel des tumeurs du pancréas exocrine inclut 

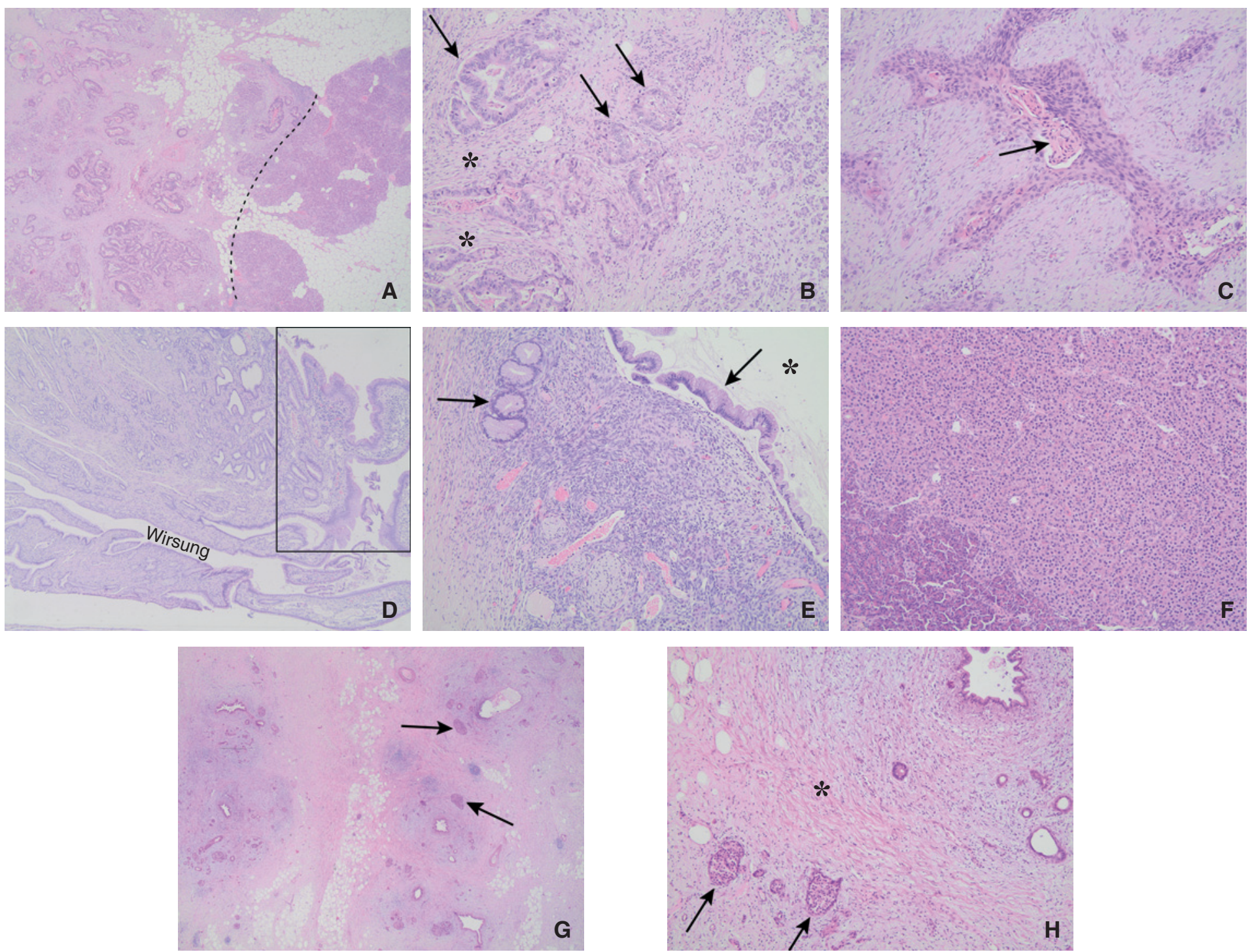

Figure 12. Sur le plan microscopique, l'aspect typique des adénocarcinomes canalaires du pancréas est celui d'une prolifération de tissu tubuliforme contrastant avec le tissu pancréatique normal (à droite de la ligne en pointillés) à faible grossissement $(\mathrm{A}, \mathrm{HE} \times 20)$. Les zones de prolifération $(\mathrm{B}$, $\mathrm{HE} \times 40$, flèches) sont associées à une fibrose desmoplasique (astérisques). Dans sa variante la plus fréquente qu'est le carcinome adénosquameux, on peut observer une maturation cornée des îlots tumoraux $(\mathrm{C}, \mathrm{HE} \times 40$, flèche). Le point de départ de la prolifération au niveau de la muqueuse duodénale $(\mathrm{D}$, $\mathrm{HE} \times 20$, encadré) ou une différenciation mucineuse $(E, H E \times 20$, flèches) des cellules tumorales au départ de la paroi d'un kyste (astérisque) signent respectivement I'ampullome et la tumeur mucineuse kystique, tandis que les tumeurs neuroendocrines sont reconnaissables à leur cellularité dense, une absence de fibrose et de structures tubulaires $(F, H E \times 20)$. Les principales affections non tumorales pouvant mimer les adénocarcinomes canalaires du pancréas sont les pancréatites chroniques dans lesquelles on n'observe pas de prolifération, mais un accroissement de la proportion d'îlots de Langerhans $(G, H E \times 20$, flèches) et une perte des lobules pancréatiques qui sont dissociés par une fibrose désorganisée $(H, H E \times 40$, astérisque).

également d'autres affections tumorales et non tumorales du pancréas et des espaces péripancréatiques qu'il est intéressant de connaître (Fig. 12).

\section{Affections tumorales}

\section{Tumeurs ampullaires et périampullaires}

Les affections tumorales simulant le plus fréquemment les adénocarcinomes céphaliques sont les tumeurs ampullaires et périampullaires, qui incluent les ampullomes, les tumeurs de la voie biliaire principale et les tumeurs duodénales périampullaires. Elles se manifestent à un stade plus précoce que les adénocarcinomes et leur opérabilité est plus fréquente. Le pronostic de ces tumeurs après procédure de Whipple est meilleur que celui des tumeurs pancréatiques céphaliques. Sur le plan anatomique, l'ampoule de Vater, qui est le canal collecteur résultant de la fusion entre les canaux biliaire et pancréatique, n'est présente que chez $60 \%$ des sujets. En son sein, les épithéliums duodénal, biliaire et pancréatique se côtoient de près. Cette particularité anatomique peut rendre difficile l'identification histologique de la tumeur, et le diagnostic définitif est souvent basé sur l'analyse morphologique de la pièce opératoire. La dilatation de la voie biliaire et/ou des canaux pancréatiques est l'anomalie la plus souvent rencontrée. La cholangiopancréatographie par IRM a progressivement remplacé la CPRE pour la mise en évidence de ces tumeurs. Les procédures endoscopiques restent cependant indiquées pour réaliser des sphinctérotomies palliatives, identifier la lésion lorsqu'aucune autre technique n'y est parvenue, et réaliser des prélèvements.

En IRM, l'ampullome apparaît comme une tumeur de petite taille entraînant une obstruction cupuliforme du bas cholédoque et faisant protrusion au sein de la lumière duodénale. Un épaississement irrégulier des parois distales du cholédoque et du canal de Wirsung peut être démontré. Rarement, les canaux pancréatiques secondaires sont dilatés, contrairement à ce qui est observé dans les tumeurs pancréatiques.

Les carcinomes de la voie biliaire distale se manifestent par une sténose progressive en sablier avec parois épaisses de la voie biliaire ou une masse endoluminale bourgeonnante rehaussée par le contraste intraveineux. Chez trois patients sur 27 (11\%) dans la série de Kim et al., une dilatation du canal de Wirsung était associée. Cette dilatation résultait d'une extension intrapancréatique 

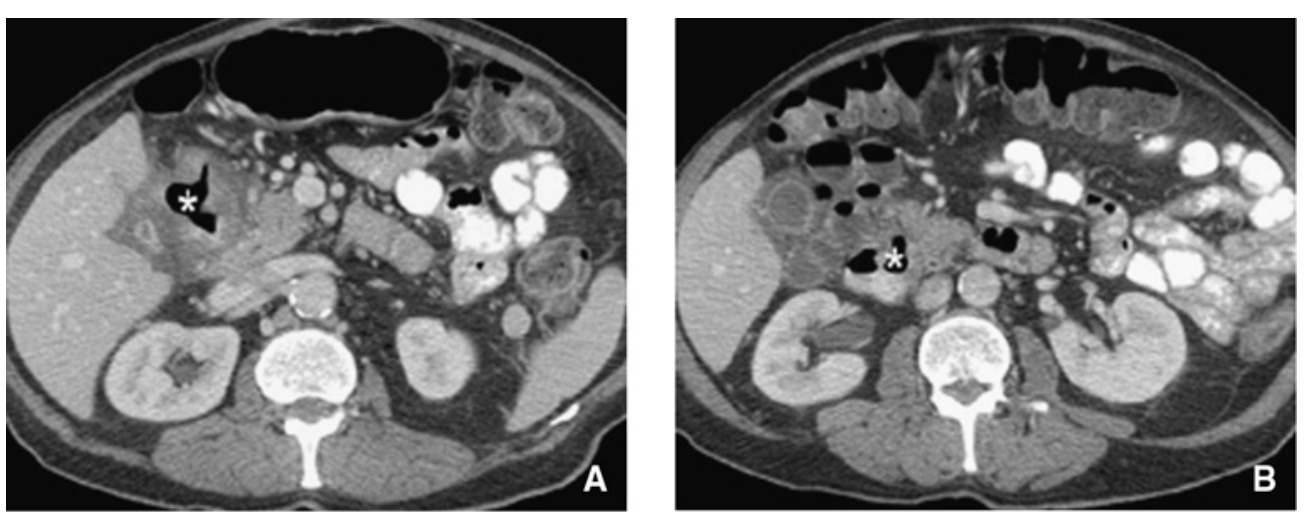

Figure 13. Tomodensitométrie axiale descendante de la tête du pancréas après contraste intraveineux. La lumière duodénale (astérisque) est délimitée de manière circonférentielle par du tissu pancréatique (flèche), permettant le diagnostic de pancréas annulaire. Noter I'œdème et l'hyperhémie des voies biliaires.
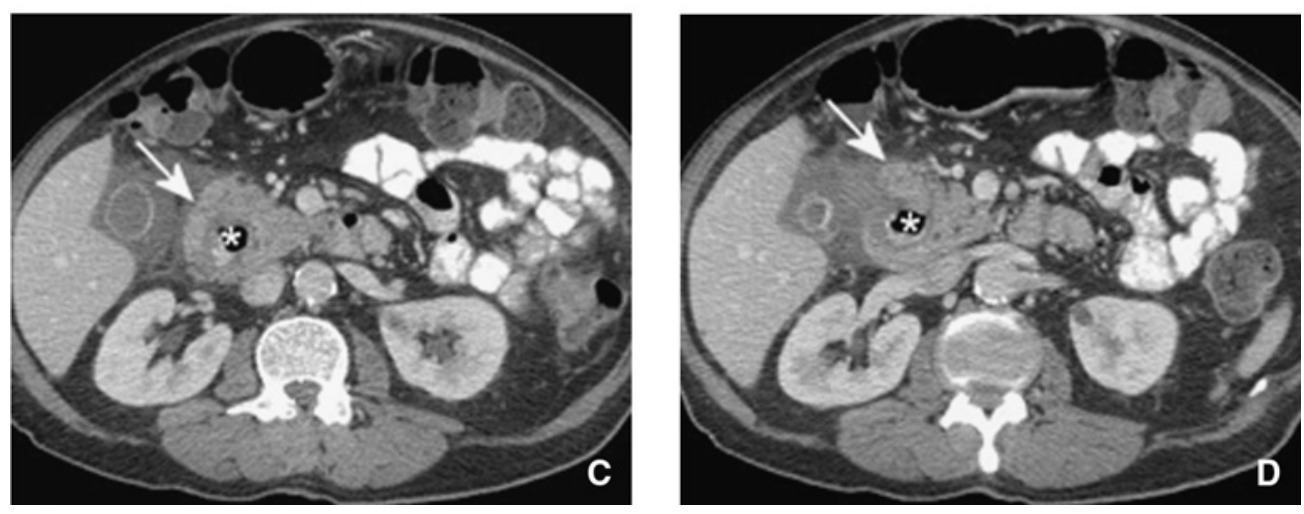

ou intra-ampullaire de la tumeur. Les adénocarcinomes duodénaux se manifestent par un épaississement de la paroi duodénale, à hauteur de la papille, avec ou sans bourgeonnement tumoral au sein de la lumière. Assez souvent, il existe une extension circonférentielle avec défaut d'expansion du duodénum et altération du relief muqueux. La dilatation des voies biliaires et des canaux pancréatiques ne survient que dans la moitié des cas.

\section{Lymphomes}

Les lymphomes de la loge pancréatique résultent le plus souvent d'une atteinte rétropéritonéale primitive. Les lymphomes pancréatiques primitifs sont rares, et le plus souvent découverts larges et infiltrants, avec d'importantes adénopathies régionales. Sur le plan de l'imagerie, la distinction entre une tumeur primitive et un envahissement secondaire peut être impossible. Les masses lymphomateuses sont le plus souvent solides mais fortement hypovasculaires; l'envahissement vasculaire est rare. De même, l'envahissement ganglionnaire en dessous du niveau des veines rénales est très rare dans les adénocarcinomes et constitue un signe distinctif avec les lymphomes ${ }^{[88]}$. Les lymphomes de type histiocytaire et le lymphome de Burkitt ont dans les séries autopsiques un tropisme pancréatique marqué, caractérisé par une atteinte dans $30 \%$ des cas pour les premiers et jusque $80 \%$ pour les seconds ${ }^{[89]}$. L'atteinte du pancréas ne modifie par ailleurs pas la prise en charge et le pronostic des sujets atteints d'un lymphome. La sarcoïdose, la maladie de Castleman et la tuberculose peuvent donner aux ganglions péripancréatiques une apparence similaire à celle de l'atteinte lymphomateuse, mais aucune de ces affections n'envahit le tissu pancréatique ${ }^{[90]}$.

\section{Métastases}

Les métastases pancréatiques ne sont pas exceptionnelles puisque, dans les séries autopsiques, elles sont retrouvées chez 3 à $12 \%$ des patients décédés d'un cancer à un stade avancé. La quasi-totalité de ces patients est également affectée par d'autres métastases extrapancréatiques ${ }^{[91]}$. Les tumeurs qui métastasent le plus souvent dans le pancréas sont les tumeurs bronchiques, mammaires, gastro-intestinales, rénales, ainsi que les mélanomes et les sarcomes.

\section{Autres tumeurs}

Les tumeurs primitives non épithéliales du pancréas sont extrêmement rares et présentes dans la littérature à titre d'observations uniques. Ces tumeurs incluent le lipome, la tumeur à cellules germinales, l'hémangiome, l'hémangioendothéliome et les sarcomes. Les tumeurs des structures adjacentes au pancréas peuvent également envahir secondairement ou simuler de manière importante les tumeurs pancréatiques primitives.

\section{Affections non tumorales}

Les affections non tumorales susceptibles de simuler les tumeurs primitives du pancréas en imagerie peuvent être subdivisées en trois principaux groupes, incluant les lésions vasculaires intra- et péripancréatiques, qui ne sont pas développées dans le détail.

\section{Variants anatomiques}

Les variants anatomiques du pancréas ne sont pas rares. À titre d'exemple, le pancréas divisum, qui résulte d'une fusion incomplète des canaux pancréatiques dorsal et ventral, peut être retrouvé chez près de $10 \%$ des sujets normaux. La relation existant entre cette anomalie et des épisodes récurrents de pancréatite aiguë est discutée dans la littérature ; en revanche, elle peut causer un élargissement de la tête du pancréas ${ }^{[92]}$. Le pancréas annulaire est en termes de fréquence la seconde anomalie congénitale du pancréas, caractérisée par un encerclement partiel ou complet du duodénum par du tissu pancréatique, susceptible en imagerie de simuler une masse de la tête du pancréas, et de causer un rétrécissement de la lumière duodénale et/ou des voies biliaires intrapancréatiques (Fig. 13). Les autres variants anatomiques cités peuvent à des degrés divers simuler des tumeurs kystiques ou solides du pancréas.

\section{Affections inflammatoires ou infectieuses}

La pancréatite chronique peut être associée à un nombre important de modifications morphologiques du pancréas. Lorsque ces changements se produisent de manière focale, ils peuvent devenir virtuellement impossibles à différencier d'un cancer, d'autant plus 

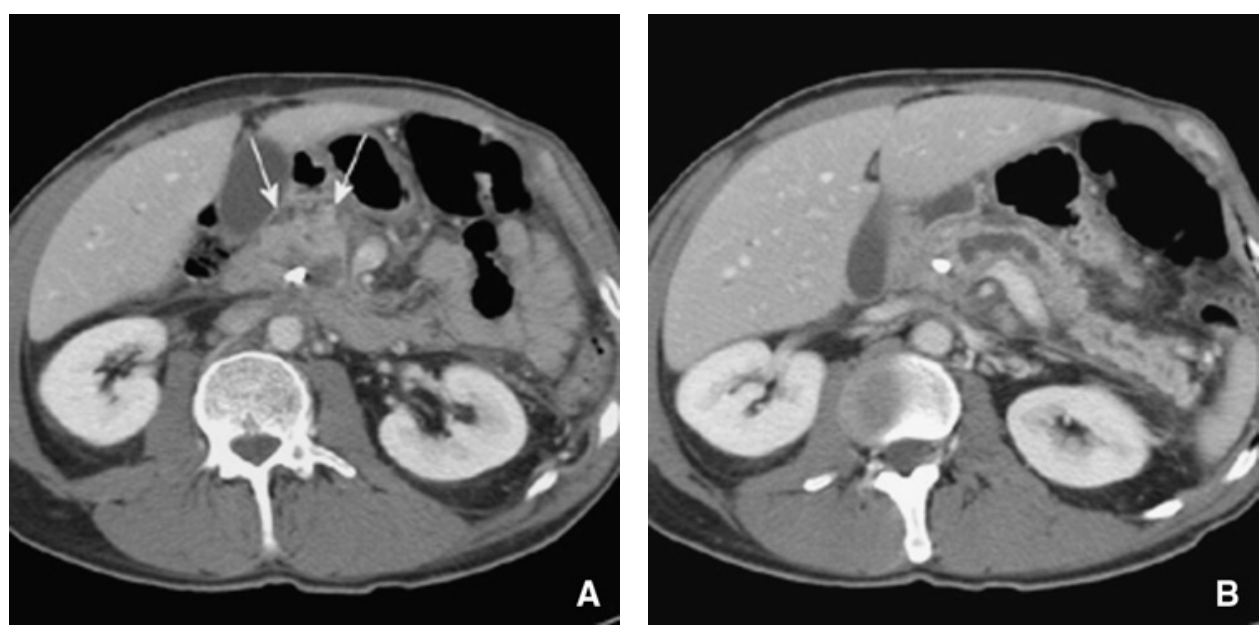

Figure 14. Tomodensitométrie axiale oblique du pancréas après contraste intraveineux. Chez ce patient, un drainage des voies biliaires a été réalisé en raison de la présence d'une masse hétérogène non calcifiée de la tête du pancréas (A) (flèches). Les canaux pancréatiques restent dilatés $(B)$. Après résection et analyse pathologique, la masse $s^{\prime}$ est révélée être un foyer de pancréatite chronique.

\section{6 Point fort}

\author{
Affections non tumorales pouvant simuler un can- \\ cer du pancréas \\ - Variants anatomiques. \\ - Pancréas divisum. \\ - Pancréas annulaire. \\ - Diverticule duodénal. \\ - Duplication duodénale. \\ - Kyste cholédocien. \\ - Involution graisseuse segmentaire. \\ - Rate accessoire, splénose, rate aberrante intrapancréa- \\ tique. \\ - Affections inflammatoires ou infectieuses. \\ - Pancréatite du sillon duodénopancréatique. \\ - Pancréatite focale. \\ - Pancréatite auto-immune (syndrome à immunoglobu- \\ lines G4). \\ - Pancréatite chronique pseudotumorale. \\ - Dystrophie kystique de pancréas aberrant. \\ - Abcès. \\ - Mucoviscidose. \\ - Lésions vasculaires. \\ - Anévrismes et pseudoanévrismes.
}

que la réaction inflammatoire qui les accompagne est en outre responsable d'une hypertrophie des ganglions régionaux et parfois d'une atteinte vasculaire (Fig. 14). Au sein de ce type d'anomalies, la pancréatite du sillon duodénopancréatique est une entité particulière se manifestant par une masse interduodénopancréatique à composante fibreuse, faiblement rehaussée par le contraste intraveineux ${ }^{[93]}$. Par ailleurs, les adénocarcinomes peuvent se développer dans le cadre d'une pancréatite chronique et les deux affections coexister; il semble ainsi exister une relation non et le cancer du pancréas ${ }^{[94]}$. Bien que de multiples techniques incluant la CPRE, la cholangiopancréatographie par IRM, la spectroscopie, la TEP et l'échoendoscopie avec biopsies ${ }^{[95-97]}$ aient été proposées pour le diagnostic différentiel, l'attitude consensuelle actuellement devant une masse du pancréas posant des problèmes diagnostiques est la résection chirurgicale lorsqu'elle est possible.

La dystrophie kystique du pancréas aberrant est associée à des épisodes récurrents de pancréatite aiguë avec formation de kystes au sein d'îlots de pancréas aberrants dans la paroi duodénale ou gastrique. Cette pathologie de fréquence inconnue est probablement sous-diagnostiquée. Elle se manifeste en imagerie par des anomalies des parois du duodénum et/ou de l'estomac qui sont encore complètement élucidée entre l'inflammation chronique épaissies, inflammatoires, et présentent en leur sein de multiples formations kystiques microscopiques et macroscopiques. Le tissu cellulograisseux périviscéral est également infiltré.

La pancréatite auto-immune se manifeste le plus souvent par une hypertrophie diffuse du tissu pancréatique, associée à des sténoses et des irrégularités de la voie biliaire intrapancréatique, et surtout des canaux pancréatiques. Une forme de cette affection peut se manifester de manière focale. L'absence de calcifications et d'envahissement vasculaire est constante. Afin d'éviter des recours indus à la chirurgie, le diagnostic doit être évoqué en présence d'un contexte clinique évocateur tel que l'existence d'une maladie auto-immune, l'absence de consommation d'alcool ${ }^{[98]}$.

Enfin, les complications locales classiques de la pancréatite aiguë, telles que l'hémorragie, le pseudokyste ou la nécrose graisseuse péripancréatique peuvent également simuler une tumeur ${ }^{[90]}$. Les infections directes de la loge pancréatique sont exceptionnelles.

\section{Conclusion}

L'adénocarcinome canalaire est la tumeur la plus fréquente du pancréas exocrine. Le seul traitement curatif demeure la résection chirurgicale. La mortalité est très élevée chez les patients non opérés. Actuellement, le détail tissulaire et la résolution spatiale offerts par les techniques d'imagerie permettent théoriquement le diagnostic précoce des adénocarcinomes du pancréas, pour autant que l'index de suspicion clinique soit élevé. La sophistication des techniques d'imagerie permet aussi, par l'évaluation des propriétés tissulaires, de restreindre le diagnostic différentiel. Enfin, la définition des critères de résécabilité des adénocarcinomes du pancréas est le domaine dans les années 2010 le plus bénéficié des progrès de l'imagerie, notamment la détection de l'envahissement vasculaire et des organes adjacents ou à distance du pancréas. Dans la pratique courante, le recours systématique à des techniques invasives d'évaluation dans ce bilan n'est plus recommandé.

Déclaration d'intérêts : les auteurs déclarent ne pas avoir de liens d'intérêts en relation avec cet article.

\section{Références}

[1] Siegel R, Ma J, Zou Z, Jemal A. Cancer statistics, 2014. CA Cancer 2014;64:9-29.

[2] Levi F, Lucchini F, Negri E, La Vecchia C. Pancreatic cancer mortality in Europe: the leveling of an epidemic. Pancreas 2003;27:139-42.

[3] Shimizu Y, Yasui K, Matsueda K, Yanagisawa A, Yamao K. Small carcinoma of the pancreas is curable: new computed tomography finding, pathological study and postoperative results from a single institute. $J$ Gastroenterol Hepatol 2005;20:1591-4. 
[4] Lynch HT, Smyrk T, Kern SE, Hruban RH, Lightdale CJ, Lemon SJ, et al. Familial pancreatic cancer: a review. Semin Oncol 1996;23:251-75.

[5] Takeshima K, Kumada T, Toyoda H, Kiriyama S, Tanikawa M, Ichikawa $\mathrm{H}$, et al. Comparison of IV contrast-enhanced sonography and histopathology of pancreatic cancer. AJR Am J Roentgenol 2005; 185:1193-200.

[6] Fusaroli P, Spada A, Mancino MG, Caletti G. Contrast harmonic echo-endoscopic ultrasound improves accuracy in diagnosis of solid pancreatic masses. Clin Gastroenterol Hepatol 2010;8 [629-34 e1-2].

[7] Tunaci M. Multidetector row CT of the pancreas. Eur J Radiol 2004; 52:18-30.

[8] Fletcher JG, Wiersema MJ, Farrell MA, Fidler JL, Burgart LJ, Koyama T, et al. Pancreatic malignancy: value of arterial, pancreatic, and hepatic phase imaging with multi-detector row CT. Radiology 2003;229:81-90.

[9] Francis IR, Cohan RH, McNulty NJ, Platt JF, Korobkin M, Gebremariam A, et al. Multidetector $\mathrm{CT}$ of the liver and hepatic neoplasms: effect of multiphasic imaging on tumor conspicuity and vascular enhancement. AJR Am J Roentgenol 2003;180:1217-24.

[10] Chung MJ, Choi BI, Han JK, Chung JW, Han MC, Bae SH. Functioning islet cell tumor of the pancreas. Localization with dynamic spiral CT. Acta Radiol 1997;38:135-8.

[11] Schima W, Fugger R, Schober E, Oettl C, Wamser P, Grabenwoger F, et al. Diagnosis and staging of pancreatic cancer: comparison of mangafodipir trisodium-enhanced MR imaging and contrast-enhanced helical hydro-CT. AJR Am J Roentgenol 2002;179:717-24.

[12] Mochizuki T, Tsukamoto E, Kuge Y, Kanegae K, Zhao S, Hikosaka $\mathrm{K}$, et al. FDG uptake and glucose transporter subtype expressions in experimental tumor and inflammation models. J Nucl Med 2001;42: $1551-5$.

[13] Wechalekar K, Sharma B, Cook G. PET/CT in oncology-a major advance. Clin Radiol 2005;60:1143-55.

[14] Rijkers AP, Valkema R, Duivenvoorden HJ, van Eijck CH. Usefulness of F-18-fluorodeoxyglucose positron emission tomography to confirm suspected pancreatic cancer: a meta-analysis. Eur J Surg Oncol 2014;40:794-804

[15] Rickes S, Unkrodt K, Neye H, Ocran KW, Wermke W. Differentiation of pancreatic tumours by conventional ultrasound, unenhanced and echo-enhanced power Doppler sonography. Scand J Gastroenterol 2002;37:1313-20.

[16] Freeny PC, Marks WM, Ryan JA, Traverso LW. Pancreatic ductal adenocarcinoma: diagnosis and staging with dynamic CT. Radiology 1988;166(1Pt1):125-33.

[17] Devesa SS, Blot WJ, Stone BJ, Miller BA, Tarone RE, Fraumeni Jr JF. Recent cancer trends in the United States. J Natl Cancer Inst 1995;87:175-82.

[18] Ahualli J. The double duct sign. Radiology 2007;244:314-5

[19] Karasawa E, Goldberg HI, Moss AA, Federle MP, London SS. CT pancreatogram in carcinoma of the pancreas and chronic pancreatitis. Radiology 1983;148:489-93.

[20] Wang ZQ, Li JS, Lu GM, Zhang XH, Chen ZQ, Meng K. Correlation of CT enhancement, tumor angiogenesis and pathologic grading of pancreatic carcinoma. World J Gastroenterol 2003;9: $2100-4$.

[21] Kang KM, Lee JM, Yoon JH, Kiefer B, Han JK, Choi BI. Intravoxel incoherent motion diffusion-weighted MR imaging for characterization of focal pancreatic lesions. Radiology 2014;270:444-53.

[22] Kitano M, Kudo M, Yamao K, Takagi T, Sakamoto H, Komaki T, et al Characterization of small solid tumors in the pancreas: the value of contrast-enhanced harmonic endoscopic ultrasonography. Am J Gastroenterol 2012;107:303-10.

[23] Edge S, Byrd DR, Compton CC, Fritz AG, Greene FL, Trotti A. Cancer staging manual. Berlin: Springer; 2010.

[24] Bipat S, Phoa SS, van Delden OM, Bossuyt PM, Gouma DJ, Lameris JS, et al. Ultrasonography, computed tomography and magnetic resonance imaging for diagnosis and determining resectability of pancreatic adenocarcinoma: a meta-analysis. J Comput Assist Tomogr 2005;29:438-45.

[25] Lytras D, Connor S, Bosonnet L, Jayan R, Evans J, Hughes M, et al. Positron emission tomography does not add to computed tomography for the diagnosis and staging of pancreatic cancer. Dig Surg 2005;22:55-61 [discussion 2].

[26] Heinrich S, Goerres GW, Schafer M, Sagmeister M, Bauerfeind P, Pestalozzi BC, et al. Positron emission tomography/computed tomography influences on the management of resectable pancreatic cancer and its cost-effectiveness. Ann Surg 2005;242:235-43.
[27] Strobel O, Hinz U, Gluth A, Hank T, Hackert T, Bergmann F, et al. Pancreatic adenocarcinoma: number of positive nodes allows to distinguish several N categories. Ann Surg 2015;261:961-9.

[28] Lu DS, Reber HA, Krasny RM, Kadell BM, Sayre J. Local staging of pancreatic cancer: criteria for unresectability of major vessels as revealed by pancreatic-phase, thin-section helical CT. AJR Am J Roentgenol 1997; 168:1439-43.

[29] Lopez Hanninen E, Amthauer H, Hosten N, Ricke J, Bohmig M, Langrehr J, et al. Prospective evaluation of pancreatic tumors: accuracy of MR imaging with MR cholangiopancreatography and MR angiography. Radiology 2002;224:34-41.

[30] Tummala P, Junaidi O, Agarwal B. Imaging of pancreatic cancer: an overview. J Gastrointest Oncol 2011;2:168-74.

[31] Brugge WR. Pancreatic fine needle aspiration: to do or not to do? JOP 2004;5:282-8.

[32] General rules for the study of pancreatic cancer. Japan Pancreas Society; 1993.

[33] Megibow AJ, Zhou XH, Rotterdam H, Francis IR, Zerhouni EA, Balfe DM, et al. Pancreatic adenocarcinoma: CT versus MR imaging in the evaluation of resectability-report of the Radiology Diagnostic Oncology Group. Radiology 1995;195:327-32.

[34] Roche CJ, Hughes ML, Garvey CJ, Campbell F, White DA, Jones L, et al. CT and pathologic assessment of prospective nodal staging in patients with ductal adenocarcinoma of the head of the pancreas. AJR Am J Roentgenol 2003;180:475-80.

[35] Muller MF, Meyenberger C, Bertschinger P, Schaer R, Marincek B. Pancreatic tumors: evaluation with endoscopic US, CT, and MR imaging. Radiology 1994;190:745-51.

[36] Midwinter MJ, Beveridge CJ, Wilsdon JB, Bennett MK, Baudouin CJ, Charnley RM. Correlation between spiral computed tomography, endoscopic ultrasonography and findings at operation in pancreatic and ampullary tumours. Br J Surg 1999;86: 189-93.

[37] Palazzo L, Roseau G, Gayet B, Vilgrain V, Belghiti J, Fekete F, et al. Endoscopic ultrasonography in the diagnosis and staging of pancreatic adenocarcinoma. Results of a prospective study with comparison to ultrasonography and CT scan. Endoscopy 1993;25: 143-50.

[38] Catalano C, Laghi A, Fraioli F, Pediconi F, Napoli A, Danti M, et al. Pancreatic carcinoma: the role of high-resolution multislice spiral CT in the diagnosis and assessment of resectability. Eur Radiol 2003:13:149-56.

[39] Irie H, Honda H, Kaneko K, Kuroiwa T, Yoshimitsu K, Masuda $\mathrm{K}$. Comparison of helical CT and MR imaging in detecting and staging small pancreatic adenocarcinoma. Abdom Imaging 1997;22: 429-33.

[40] Motosugi U, Ichikawa T, Morisaka H, Sou H, Muhi A, Kimura $\mathrm{K}$, et al. Detection of pancreatic carcinoma and liver metastases with gadoxetic acid-enhanced MR imaging: comparison with contrast-enhanced multi-detector row CT. Radiology 2011;260: 446-53.

[41] Kauhanen SP, Komar G, Seppanen MP, Dean KI, Minn HR, Kajander SA, et al. A prospective diagnostic accuracy study of 18 F-fluorodeoxyglucose positron emission tomography/computed tomography, multidetector row computed tomography, and magnetic resonance imaging in primary diagnosis and staging of pancreatic cancer. Ann Surg 2009;250:957-63.

[42] Sahani DV, Kalva SP, Fischman AJ, Kadavigere R, Blake M, Hahn $\mathrm{PF}$, et al. Detection of liver metastases from adenocarcinoma of the colon and pancreas: comparison of mangafodipir trisodiumenhanced liver MRI and whole-body FDG PET. AJR Am J Roentgenol 2005; 185:239-46

[43] Ellsmere J, Mortele K, Sahani D, Maher M, Cantisani V, Wells W, et al. Does multidetector-row CT eliminate the role of diagnostic laparoscopy in assessing the resectability of pancreatic head adenocarcinoma? Surg Endosc 2005;19:369-73.

[44] Kloppel G, Maillet B. Classification and staging of pancreatic nonendocrine tumors. Radiol Clin North Am 1989;27:105-19.

[45] Parkin DM, Pisani P, Ferlay J. Estimates of the worldwide incidence of eighteen major cancers in 1985. Int J Cancer 1993;54: 594-606.

[46] Ryan DP, Hong TS, Bardeesy N. Pancreatic adenocarcinoma. N Engl J Med 2014;371:2140-1.

[47] Vasen HF, Wasser M, van Mil A, Tollenaar RA, Konstantinovski M, Gruis NA, et al. Magnetic resonance imaging surveillance detects early-stage pancreatic cancer in carriers of a p16-Leiden mutation. Gastroenterology 2011;140:850-6. 
[48] Verna EC, Hwang C, Stevens PD, Rotterdam H, Stavropoulos SN, Sy $\mathrm{CD}$, et al. Pancreatic cancer screening in a prospective cohort of highrisk patients: a comprehensive strategy of imaging and genetics. Clin Cancer Res 2010;16:5028-37.

[49] Brentnall TA, Bronner MP, Byrd DR, Haggitt RC, Kimmey MB. Early diagnosis and treatment of pancreatic dysplasia in patients with a family history of pancreatic cancer. Ann Intern Med 1999;131:247-55.

[50] Steinberg WM, Barkin JS, Bradley 3rd EL, DiMagno E, Layer P, Canto MI, et al. Should patients with a strong family history of pancreatic cancer be screened on a periodic basis for cancer of the pancreas? Pancreas 2009;38: 137-50.

[51] Grover BT, Gundersen 3rd SB, Kothari SN. Video. Laparoscopic distal pancreatectomy and splenectomy for splenic artery aneurysm. Surg Endosc 2010;24:2318-20.

[52] Templeton AW, Brentnall TA. Screening and surgical outcomes of familial pancreatic cancer. Surg Clin North Am 2013;93:629-45.

[53] Kalser MH, Barkin J, MacIntyre JM. Pancreatic cancer. Assessment of prognosis by clinical presentation. Cancer 1985;56:397-402.

[54] Mannell A, van Heerden JA, Weiland LH, Ilstrup DM. Factors influencing survival after resection for ductal adenocarcinoma of the pancreas. Ann Surg 1986;203:403-7.

[55] Palsson B, Masson P, Andren-Sandberg A. Tumour marker CA 50 levels compared to signs and symptoms in the diagnosis of pancreatic cancer. Eur J Surg Oncol 1997;23:151-6.

[56] Moossa AR, Levin B. The diagnosis of "early" pancreatic cancer: the University of Chicago experience. Cancer 1981;47(Suppl. 6):1688-97.

[57] Pleskow DK, Berger HJ, Gyves J, Allen E, McLean A, Podolsky DK. Evaluation of a serologic marker. CA19-9, in the diagnosis of pancreatic cancer. Ann Intern Med 1989;110:704-9.

[58] Safi F, Schlosser W, Falkenreck S, Beger HG. CA 19-9 serum course and prognosis of pancreatic cancer. Int J Pancreatol 1996;20:155-61.

[59] Gandolfi L, Torresan F, Solmi L, Puccetti A. The role of ultrasound in biliary and pancreatic diseases. Eur J Ultrasound 2003;16:141-59.

[60] Pollock D, Taylor KJ. Ultrasound scanning in patients with clinical suspicion of pancreatic cancer: a retrospective study. Cancer 1981;47(Suppl. 6):1662-5.

[61] Pandharipande PV, Heberle C, Dowling EC, Kong CY, Tramontano A, Perzan KE, et al. Targeted screening of individuals at high risk for pancreatic cancer: results of a simulation model. Radiology 2015;275:177-87.

[62] Brand B, Pfaff T, Binmoeller KF, Sriram PV, Fritscher-Ravens A, Knofel WT, et al. Endoscopic ultrasound for differential diagnosis of focal pancreatic lesions, confirmed by surgery. Scand J Gastroenterol 2000;35:1221-8

[63] Sendler A, Avril N, Helmberger H, Stollfuss J, Weber W, Bengel $\mathrm{F}$, et al. Preoperative evaluation of pancreatic masses with positron emission tomography using $18 \mathrm{~F}$-fluorodeoxyglucose: diagnostic limitations. World J Surg 2000;24:1121-9.

[64] Trede M, Schwall G, Saeger HD. Survival after pancreatoduodenectomy. 118 consecutive resections without an operative mortality. Ann Surg 1990;211:447-58.

[65] Jimenez RE, Warshaw AL, Rattner DW, Willett CG, McGrath D, Fernandez-del Castillo C. Impact of laparoscopic staging in the treatment of pancreatic cancer. Arch Surg 2000;135:409-14 [discussion 14-5].

[66] Polati E, Finco G, Gottin L, Bassi C, Pederzoli P, Ischia S. Prospective randomized double-blind trial of neurolytic coeliac plexus block in patients with pancreatic cancer. Br J Surg 1998;85:199-201.

[67] Speer AG, Cotton PB, Russell RC, Mason RR, Hatfield AR, Leung JW, et al. Randomised trial of endoscopic versus percutaneous stent insertion in malignant obstructive jaundice. Lancet 1987;2:57-62.

[68] Prat F, Chapat O, Ducot B, Ponchon T, Pelletier G, Fritsch J, et al A randomized trial of endoscopic drainage methods for inoperable malignant strictures of the common bile duct. Gastrointest Endosc 1998;47:1-7

[69] Singh SM, Longmire Jr WP, Reber HA. Surgical palliation for pancreatic cancer. The UCLA experience. Ann Surg 1990;212:132-9.

[70] Rombouts SJ, Vogel JA, van Santvoort HC, van Lienden KP, van Hillegersberg R, Busch OR, et al. Systematic review of innovative ablative therapies for the treatment of locally advanced pancreatic cancer. $\mathrm{Br} J$ Surg 2015;102:182-93.

[71] Stumpp P, Kloppel R, Kahn T. Imaging after a whipple operation: improving visibility of the afferent jejunal loop in spiral computed tomography using biliary contrast medium. J Comput Assist Tomogr 2005;29:394-400.

[72] Ruf J, Lopez Hanninen E, Oettle H, Plotkin M, Pelzer U, Stroszczynski $\mathrm{C}$, et al. Detection of recurrent pancreatic cancer: comparison of FDGPET with CT/MRI. Pancreatology 2005;5:266-72.
[73] Sperti C, Pasquali C, Bissoli S, Chierichetti F, Liessi G, Pedrazzoli S. Tumor relapse after pancreatic cancer resection is detected earlier by 18-FDG PET than by CT. J Gastrointest Surg 2010;14: 131-40.

[74] Casneuf V, Delrue L, Kelles A, Van Damme N, Van Huysse J, Berrevoet $\mathrm{F}$, et al. Is combined $18 \mathrm{~F}$-fluorodeoxyglucose-positron emission tomography/computed tomography superior to positron emission tomography or computed tomography alone for diagnosis, staging and restaging of pancreatic lesions? Acta Gastroenterol Belg 2007;70:331-8.

[75] Friedman AC, Edmonds PR. Rare pancreatic malignancies. Radiol Clin North Am 1989;27:177-90.

[76] Chiou YY, Chiang JH, Hwang JI, Yen CH, Tsay SH, Chang CY. Acinar cell carcinoma of the pancreas: clinical and computed tomography manifestations. J Comput Assist Tomogr 2004;28:180-6.

[77] Tatli S, Mortele KJ, Levy AD, Glickman JN, Ros PR, Banks PA, et al. $\mathrm{CT}$ and MRI features of pure acinar cell carcinoma of the pancreas in adults. AJR Am J Roentgenol 2005;184:511-9.

[78] Holen KD, Klimstra DS, Hummer A, Gonen M, Conlon K, Brennan $\mathrm{M}$, et al. Clinical characteristics and outcomes from an institutional series of acinar cell carcinoma of the pancreas and related tumors. $J$ Clin Oncol 2002;20:4673-8.

[79] Friedman AC, Lichtenstein JE, Fishman EK, Oertel JE, Dachman AH, Siegelman SS. Solid and papillary epithelial neoplasm of the pancreas. Radiology 1985;154:333-7.

[80] Cantisani V, Mortele KJ, Levy A, Glickman JN, Ricci P, Passariello $\mathrm{R}$, et al. MR imaging features of solid pseudopapillary tumor of the pancreas in adult and pediatric patients. AJR Am J Roentgenol 2003;181:395-401.

[81] Montemarano H, Lonergan GJ, Bulas DI, Selby DM. Pancreatoblastoma: imaging findings in 10 patients and review of the literature. Radiology 2000;214:476-82.

[82] Klimstra DS, Wenig BM, Adair CF, Heffess CS. Pancreatoblastoma. A clinicopathologic study and review of the literature. Am J Surg Pathol 1995:19:1371-89.

[83] Rosebrook JL, Glickman JN, Mortele KJ. Pancreatoblastoma in an adult woman: sonography, CT, and dynamic gadolinium-enhanced MRI features. AJR Am J Roentgenol 2005;184(Suppl. 3):S78-81.

[84] Kohda E, Iseki M, Ikawa H, Endoh M, Yokoyama J, Mukai M, et al. Pancreatoblastoma. Three original cases and review of the literature. Acta Radiol 2000;41:334-7.

[85] Demos TC, Posniak HV, Harmath C, Olson MC, Aranha G. Cystic lesions of the pancreas. AJR Am J Roentgenol 2002;179:1375-88.

[86] Cohen-Scali F, Vilgrain V, Brancatelli G, Hammel P, Vullierme MP, Sauvanet A, et al. Discrimination of unilocular macrocystic serous cystadenoma from pancreatic pseudocyst and mucinous cystadenoma with CT: initial observations. Radiology 2003;228:727-33.

[87] Visser BC, Muthusamay VR, Mulvihill SJ, Coakley F. Diagnostic imaging of cystic pancreatic neoplasms. Surg Oncol 2004;13: 27-39.

[88] Merkle EM, Bender GN, Brambs HJ. Imaging findings in pancreatic lymphoma: differential aspects. AJR Am J Roentgenol 2000; 174:671-5.

[89] Graif M, Kessler A, Neumann Y, Martinowitz U, Itzchak Y. Pancreatic Burkitt lymphoma in AIDS: sonographic appearance. AJR Am J Roentgenol 1987;149:1290-1.

[90] To'o KJ, Raman SS, Yu NC, Kim YJ, Crawford T, Kadell BM, et al. Pancreatic and peripancreatic diseases mimicking primary pancreatic neoplasia. Radiographics 2005;25:949-65.

[91] Adsay NV, Andea A, Basturk O, Kilinc N, Nassar H, Cheng JD. Secondary tumors of the pancreas: an analysis of a surgical and autopsy database and review of the literature. Virchows Archiv 2004:444:527-35

[92] Soulen MC, Zerhouni EA, Fishman EK, Gayler BW, Milligan F, Siegelman SS. Enlargement of the pancreatic head in patients with pancreas divisum. Clin Imaging 1989;13:51-7.

[93] Irie H, Honda H, Kuroiwa T, Hanada K, Yoshimitsu K, Tajima T, et al. MRI of groove pancreatitis. J Comput Assist Tomogr 1998;22:651-5.

[94] Whitcomb DC. Inflammation and Cancer V. Chronic pancreatitis and pancreatic cancer. Am J Physiol Gastrointest Liver Physiol 2004;287:G315-9.

[95] Yusuf TE, Bhutani MS. Differentiating pancreatic cancer from pseudotumorous chronic pancreatitis. Curr Gastroenterol Rep 2002;4:135-9.

[96] Cho SG, Lee DH, Lee KY, Ji H, Lee KH, Ros PR, et al. Differentiation of chronic focal pancreatitis from pancreatic carcinoma by in vivo proton magnetic resonance spectroscopy. J Comput Assist Tomogr 2005;29:163-9. 
[97] Bang S, Chung HW, Park SW, Chung JB, Yun M, Lee JD, et al. The clinical usefulness of 18-fluorodeoxyglucose positron emission tomography in the differential diagnosis, staging, and response evaluation after concurrent chemoradiotherapy for pancreatic cancer. $J$ Clin Gastroenterol 2006;40:923-9.
[98] Wakabayashi T, Kawaura Y, Satomura Y, Watanabe H, Motoo Y, Okai $\mathrm{T}$, et al. Clinical and imaging features of autoimmune pancreatitis with focal pancreatic swelling or mass formation: comparison with so-called tumor-forming pancreatitis and pancreatic carcinoma. Am J Gastroenterol 2003;98:2679-87.

U. Duran.

Service de radiodiagnostic, Domaine universitaire du Sart-Tilman B35, 4000 Liège, Belgique.

D. Brisbois.

R. Materne.

Service de radiologie, Centre hospitalier Chrétien, rue de Hesbaye, 75, 4000 Liège, Belgique.

C. Tchuisse Noukoua.

Service de radiodiagnostic, Domaine universitaire du Sart-Tilman B35, 4000 Liège, Belgique.

N. Blétard.

E. Mutijima.

Service d'anatomie pathologique, Domaine universitaire du Sart-Tilman B35, 4000 Liège, Belgique.

A. Nchimi (anchimi@chu.ulg.ac.be).

Service de radiodiagnostic, Domaine universitaire du Sart-Tilman B35, 4000 Liège, Belgique.

Toute référence à cet article doit porter la mention : Duran U, Brisbois D, Materne R, Tchuisse Noukoua C, Blétard N, Mutijima E, et al. Cancers du pancréas exocrine. EMC - Radiologie et imagerie médicale - abdominale - digestive 2015;0(0):1-20 [Article 33-653-A-10].

\section{Disponibles sur www.em-consulte.com}

